\title{
S-expansions in dimension two
}

\author{
par BERNHARD SCHRATZBERGER
}

\begin{abstract}
RÉSUMÉ. Nous généralisons en dimension deux la méthode de singularisation développée par C. Kraikamp au cours des années 90 dans ses travaux sur les systèmes dynamiques associées aux fractions continues, en relation avec certaines propriétés d'approximations diophantiennes. Nous appliquons la méthode à l'algorithme de Brun en dimension 2 et montrons comment utiliser cette technique et d'autres analogues pour transférer des propriétés métriques et diophantiennes d'un algorithme à l'autre. Une conséquence de cette étude est la construction d'un algorithme qui améliore les propriétés d'approximations par comparaisons avec celles de l'algorithme de Brun.
\end{abstract}

ABSTRACT. The technique of singularization was developped by C. Kraaikamp during the nineties, in connection with his work on dynamical systems related to continued fraction algorithms and their diophantine approximation properties. We generalize this technique from one into two dimensions. We apply the method to the the two dimensional Brun's algorithm. We discuss, how this technique, and related ones, can be used to transfer certain metrical and diophantine properties from one algorithm to the others. In particular, we are interested in the transferability of the density of the invariant measure. Finally, we use this method to construct an algorithm which improves approximation properties, as opposed to Brun's algorithm.

\section{Introduction}

The technique of singularization, as described in details by M. Iosifescu and C. Kraaikamp [7] (see also [9]), was introduced to improve some diophantine approximation properties of the regular one-dimensional continued fraction algorithm in the following sense: Let $\left\{p^{(t)} / q^{(t)}\right\}_{t=1}^{\infty}$ be the sequence of convergents of an arbitrary real number $x$ in $(0,1)$, produced by the regular continued fraction algorithm. Singularization methods allow to transform the original (regular continued fraction) algorithm into new ones (depending on the actual setting of the applications), such that the

Manuscrit reçu le 5 juin 2003. 
sequences of convergents built from the new algorithms are subsequences of the previous one. This technique also allows to transfer the underlying ergodic properties of one algorithm to the other.

A large family of semi-regular continued fraction algorithms, called $S$ expansions, can be related to each other via singularizations (e.g. the nearest integer continued fraction [14], Hurwitz' singular continued fraction [6], Minkowski's diagonal expansion [13], Nakada's $\alpha$-expansions $[15,16]$ or Bosma's optimal continued fraction [2]).

In this paper, we show that similar techniques can be applied in dimension two. We describe singularization processes, based on the twodimensional Brun's algorithm, and analyze how to use singularizations to transfer certain statistical and approximation properties towards the resulting algorithm. In particular, using natural extensions of the underlying ergodic dynamical systems, we are interested in how to deduce the corresponding invariant measure of the new algorithms from the density of the invariant measure of the original algorithm. This is of a special interest with respect to recent investigations by the author on similar relations between Brun's algorithm and the Jacobi-Perron algorithm in two dimensions [19] .

Finally, we present an algorithm $\bar{T}_{q}$ with improved approximation properties, as opposed to the underlying Brun's algorithm.

\section{Definitions}

We recall some basic definitions and results on fibered systems. For an extensive summary, we refer to a monograph of F. Schweiger [23].

Definition. Let $X$ be a set and $T: X \rightarrow X$. If there exists a partition $\{X(i): i \in I\}$ of $X$, where $I$ is finite or countable, such that the restriction of $T$ to $X(i)$ is injective, then $(X, T)$ is called a fibred system.

$I$ is the set of digits, and the partition $\{X(i): i \in I\}$ is called the time-1-partition.

Definition. A cylinder of rank $t$ is the set

$$
X\left(i^{(1)}, \ldots, i^{(t)}\right):=\left\{x: i(x)=i^{(1)}, \ldots, i\left(T^{t-1}(x)\right)=i^{(t)}\right\} .
$$

A block of digits $\left(i^{(1)}, \ldots, i^{(t)}\right)$ is called admissible, if

$$
X\left(i^{(1)}, \ldots, i^{(t)}\right) \neq \emptyset .
$$

Since $T: X(i) \rightarrow T X(i)$ is bijective, there exists an inverse map $V(i)$ : $T X(i) \rightarrow X(i)$ which will be called a local inverse branch of $T$. Define $V\left(i^{(1)}, i^{(2)}, \ldots, i^{(t)}\right):=V\left(i^{(1)}\right) \circ V\left(i^{(2)}, \ldots, i^{(t)}\right)$; then $V\left(i^{(1)}, i^{(2)}, \ldots, i^{(t)}\right)$ is a local inverse branch of $T^{t}$. 
Definition. The fibred system $(X, T)$ is called a multidimensional continued fraction algorithm if

(1) $X$ is a subset of the Euclidean space $\mathbb{R}^{n}$

(2) For every digit $i \in I$, there is an invertible matrix $\alpha=\alpha(i)=\left(\left(a_{k l}\right)\right)$, $0 \leq k, l \leq n$, such that $x^{(1)}=T x^{(0)}, x^{(0)} \in X$, is given as

$$
x_{k}^{(1)}=\frac{a_{k 0}+\sum_{l=1}^{n} a_{k l} x_{l}^{(0)}}{a_{00}+\sum_{l=1}^{n} a_{0 l} x_{l}^{(0)}} .
$$

In this paper, the process of singularization will be applied to the following algorithm:

Definition (Brun 1957). Let $M:=\left\{\left(b_{0}, b_{1}, b_{2}\right): b_{0} \geq b_{1} \geq b_{2} \geq 0\right\}$. Brun's Algorithm is generated by the map $\tau_{S}: M \rightarrow M$, where

$$
\tau_{S}\left(b_{0}, b_{1}, b_{2}\right)=\left\{\begin{array}{lll}
\left(b_{0}-b_{1}, b_{1}, b_{2}\right), & b_{0}-b_{1} \geq b_{1} & (j=0), \\
\left(b_{1}, b_{0}-b_{1}, b_{2}\right), & b_{1} \geq b_{0}-b_{1} \geq b_{2} & (j=1), \\
\left(b_{1}, b_{2}, b_{0}-b_{1}\right), & b_{2} \geq b_{0}-b_{1} & (j=2) .
\end{array}\right.
$$

Let $X_{B}:=\left\{\left(x_{1}, x_{2}\right): 1 \geq x_{1} \geq x_{2} \geq 0\right\}$; using the projection map $p: M \rightarrow X_{B}$, defined by

$$
p\left(b_{0}, b_{1}, b_{2}\right)=\left(\frac{b_{1}}{b_{0}}, \frac{b_{2}}{b_{0}}\right)
$$

we obtain the corresponding two-dimensional map $T_{S}: X_{B} \rightarrow X_{B}$,

$$
T_{S}\left(x_{1}, x_{2}\right)=\left\{\begin{array}{lll}
\left(\frac{x_{1}}{1-x_{1}}, \frac{x_{2}}{1-x_{1}}\right), & 1-x_{1} \geq x_{1} & (j=0), \\
\left(\frac{1-x_{1}}{x_{1}}, \frac{x_{2}}{x_{1}}\right), & x_{1} \geq 1-x_{1} \geq x_{2} & (j=1), \\
\left(\frac{x_{2}}{x_{1}}, \frac{1-x_{1}}{x_{1}}\right), & x_{2} \geq 1-x_{1} & (j=2) .
\end{array}\right.
$$

We refer to $j$ as the type of the algorithm. Denote

$$
\begin{aligned}
& X_{B}(0):=\left\{\left(x_{1}, x_{2}\right) \in X_{B}: j\left(x_{1}, x_{2}\right)=0\right\} \\
& X_{B}(1):=\left\{\left(x_{1}, x_{2}\right) \in X_{B}: j\left(x_{1}, x_{2}\right)=1\right\} \\
& X_{B}(2):=\left\{\left(x_{1}, x_{2}\right) \in X_{B}: j\left(x_{1}, x_{2}\right)=2\right\}
\end{aligned}
$$

Further, for $t \geq 1$, define $j^{(t)}=j^{(t)}\left(x_{1}^{(0)}, x_{2}^{(0)}\right):=j\left(T_{S}^{t-1}\left(x_{1}^{(0)}, x_{2}^{(0)}\right)\right)$. The cylinders $X_{B}\left(j^{(1)}, \ldots, j^{(t)}\right)$ of the fibred system $\left(X_{B}, T_{S}\right)$ are full, i.e., $T_{S}^{t} X_{B}\left(j^{(1)}, \ldots, j^{(t)}\right)=X_{B}$. The algorithm is ergodic and conservative with respect to Lebesgue measure (see Theorem 21 in [23], p. 50).

Let $t \geq 1$; the matrices $\alpha_{S}^{(t)}:=\alpha_{S}\left(j^{(t)}\right)$ of Brun's Algorithm are given as

$$
\alpha_{S}(0)=\left(\begin{array}{ccc}
1 & -1 & 0 \\
0 & 1 & 0 \\
0 & 0 & 1
\end{array}\right), \alpha_{S}(1)=\left(\begin{array}{ccc}
0 & 1 & 0 \\
1 & -1 & 0 \\
0 & 0 & 1
\end{array}\right), \alpha_{S}(2)=\left(\begin{array}{ccc}
0 & 1 & 0 \\
0 & 0 & 1 \\
1 & -1 & 0
\end{array}\right) \text {. }
$$




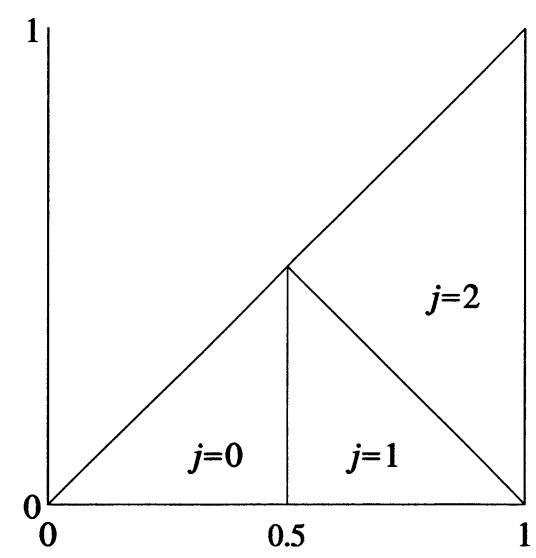

Figure 1. The time-1-partition of Brun's Algorithm $T_{S}$

The inverses $\beta_{S}^{(t)}:=\beta_{S}\left(j^{(t)}\right)$ of the matrices of the algorithm with

$$
\beta_{S}(0)=\left(\begin{array}{lll}
1 & 1 & 0 \\
0 & 1 & 0 \\
0 & 0 & 1
\end{array}\right), \quad \beta_{S}(1)=\left(\begin{array}{lll}
1 & 1 & 0 \\
1 & 0 & 0 \\
0 & 0 & 1
\end{array}\right), \quad \beta_{S}(2)=\left(\begin{array}{lll}
1 & 0 & 1 \\
1 & 0 & 0 \\
0 & 1 & 0
\end{array}\right),
$$

produce a sequence of convergence matrices $\left\{\Omega_{S}^{(s)}\right\}_{s=0}^{\infty}$ as follows:

Definition. Let $E$ be the identity matrix. Then

$$
\begin{gathered}
\Omega_{S}^{(0)}=\left(\begin{array}{lll}
q^{(0)} & q^{\left(0^{\prime}\right)} & q^{\left(0^{\prime \prime}\right)} \\
p_{1}^{(0)} & p_{1}^{\left(0^{\prime}\right)} & p_{1}^{\left(0^{\prime \prime}\right)} \\
p_{2}^{(0)} & p_{2}^{\left(0^{\prime}\right)} & p_{2}^{\left(0^{\prime \prime}\right)}
\end{array}\right):=E \\
\Omega_{S}^{(t)}=\left(\begin{array}{lll}
q^{(t)} & q^{\left(t^{\prime}\right)} & q^{\left(t^{\prime \prime}\right)} \\
p_{1}^{(t)} & p_{1}^{\left(t^{\prime}\right)} & p_{1}^{\left(t^{\prime \prime}\right)} \\
p_{2}^{(t)} & p_{2}^{\left(t^{\prime}\right)} & p_{2}^{\left(t^{\prime \prime}\right)}
\end{array}\right):=\Omega_{S}^{(t-1)} \beta_{S}^{(t)} .
\end{gathered}
$$

Hence, for $k=1,2,\left(x_{1}^{(t)}, x_{2}^{(t)}\right)=T_{S}^{t}\left(x_{1}^{(0)}, x_{2}^{(0)}\right)$,

$$
x_{k}^{(0)}=\frac{p_{k}^{(t)}+x_{1}^{(t)} p_{k}^{\left(t^{\prime}\right)}+x_{2}^{(t)} p_{k}^{\left(t^{\prime \prime}\right)}}{q^{(t)}+x_{1}^{(t)} q^{\left(t^{\prime}\right)}+x_{2}^{(t)} q^{\left(t^{\prime \prime}\right)}} .
$$

The columns of the convergence matrices produce Diophantine approximations $\left(p_{1}^{(t)} / q^{(t)}, p_{2}^{(t)} / q^{(t)}\right)$ to $\left(x_{1}^{(0)}, x_{2}^{(0)}\right)$. Similar to the above, $j$ is referred to as the type of a matrix $\beta_{S}(j)$

\section{The process of Singularization}

The basic idea of singularization, as introduced by C. Kraaikamp [9], was to improve approximation properties of the (one-dimensional) regular continued fraction algorithm. In particular, C. Kraaikamp was interested in 
semi-regular continued fraction algorithms, whose sequences of convergents $\left\{p^{(t)} / q^{(t)}\right\}_{t=1}^{\infty}$ were subsequences of the sequence of regular convergents of $x$. To construct these algorithms, he introduced the process of singularization, which further led to the definition of a new class of semi-regular continued fraction algorithms, the $S$-expansions.

The process is defined by a law of singularization which, to a given continued fraction algorithm (or a class of such algorithms), determines in an unambiguous way the convergents to be singularized by using some specific matrix identities.

We give an example for Brun's Algorithm in two dimensions. The following matrix identities are easily checked (for an arbitrary $t, \phi_{t}$ and $\psi_{t}$ are defined such that either $\phi_{t}=1$ and $\psi_{t}=0$, or $\phi_{t}=0$ and $\psi_{t}=1$ ):

type $M_{1}$ :

$$
\left(\begin{array}{ccc}
1 & A_{1} & 0 \\
0 & 1 & 0 \\
0 & 0 & 1
\end{array}\right)\left(\begin{array}{ccc}
1 & A_{2} & 0 \\
0 & 1 & 0 \\
0 & 0 & 1
\end{array}\right)=\left(\begin{array}{ccc}
1 & A_{1}+A_{2} & 0 \\
0 & 1 & 0 \\
0 & 0 & 1
\end{array}\right),
$$

type $M_{2}$ :

$$
\left(\begin{array}{ccc}
1 & A_{1} & 0 \\
0 & 1 & 0 \\
0 & 0 & 1
\end{array}\right)\left(\begin{array}{ccc}
A_{2} & \phi_{2} & \psi_{2} \\
1 & 0 & 0 \\
0 & \psi_{2} & \phi_{2}
\end{array}\right)=\left(\begin{array}{ccc}
A_{1}+A_{2} & \phi_{2} & \psi_{2} \\
1 & 0 & 0 \\
0 & \psi_{2} & \phi_{2}
\end{array}\right) .
$$

Based on these identities, we remove any matrix $\beta_{S}^{(t)}$ from the sequence of inverse matrices if $j^{(t)}=0$. The matrix $\beta^{(t+1)}$ should then be replaced according to the above rule. Thus a new sequence of convergence matrices $\left\{\Omega_{S}^{*(s)}\right\}_{s=0}^{\infty}$ is obtained by removing $\Omega_{S}^{(t)}$ from $\left\{\Omega_{S}^{(s)}\right\}_{s=0}^{\infty}$. Clearly, the sequence of Diophantine approximations $\left\{\left(p_{1}^{*(s)} / q^{*(s)}, p_{2}^{*(s)} / q^{*(s)}\right)\right\}_{s=0}^{\infty}$ obtained from the new convergence matrices is a subsequence of the original one.

Now we apply the same procedure to any remaining matrix of type 0 , and continue until all such matrices have been removed. That way, a new algorithm is defined. This transformation of the original algorithm into a new one is called a singularization. We put this into a more general form:

Definition. A transformation $\sigma_{t}$, defined by a matrix identity that removes the matrix $\beta^{(t)}$ from the sequence of inverse matrices (which changes an algorithm into a new form such that the sequence of Diophantine approximations $\left\{\left(p_{1}^{*(s)} / q^{*(s)}, p_{2}^{*(s)} / q^{*(s)}\right)\right\}_{s=0}^{\infty}$ obtained from the new algorithm is a subsequence of the original one) is called a singularization. We say we have singularized the matrix $\beta^{(t)}$.

By the definition, the sequence of convergents of the singularized algorithm is a subsequence of the sequence of convergents of the original one. Therefore, if the original algorithm converges to $\left(x_{1}, x_{2}\right)$ so does the new one. Now we define the exponent of convergence as the supremum of real 
numbers $d$ such that for almost all $\left(x_{1}, x_{2}\right)$ and all $t$ large enough, the inequalities

$$
\left|x_{k}-\frac{p_{k}^{(t)}}{q^{(t)}}\right| \leq \frac{1}{\left(q^{(t)}\right)^{1+d}} \quad(k=1,2)
$$

hold. Notice that the exponent of convergence of the singularized algorithm is always larger or equal to the one of the original algorithm.

We generalize the definition of matrices $\beta_{S}(j), j=0,1,2$, to

$$
\begin{gathered}
\beta_{M}(0, A)=\left(\begin{array}{ccc}
1 & A & 0 \\
0 & 1 & 0 \\
0 & 0 & 1
\end{array}\right), \beta_{M}(1, A)=\left(\begin{array}{ccc}
A & 1 & 0 \\
1 & 0 & 0 \\
0 & 0 & 1
\end{array}\right), \\
\beta_{M}(2, A)=\left(\begin{array}{ccc}
A & 0 & 1 \\
1 & 0 & 0 \\
0 & 1 & 0
\end{array}\right) .
\end{gathered}
$$

We may thus define a law of singularization $\mathrm{LM}^{*}$ for Brun's Algorithm, to obtain its multiplicative acceleration $T_{M}$ (a different, but equivalent rule LM will be introduced Section 4).

Law of singularization LM*: Singularize every matrix $\beta_{M}(0, A)$, using identities $M_{1}$ and $M_{2}$.

Consider a block of successive matrices of type 0 . Note that matrix identities $M_{1}$ and $M_{2}$ allow singularizations of these matrices in an arbitrary order, yielding the same algorithm, as long as we remove every such matrix.

The resulting algorithm is the well-known multiplicative acceleration of Brun's Algorithm $T_{M}: X_{B} \rightarrow X_{B}$,

$$
T_{M}\left(x_{1}, x_{2}\right)=\left\{\begin{array}{lll}
\left(\frac{1}{x_{1}}-A, \frac{x_{2}}{x_{1}}\right), & \frac{1}{x_{1}}-A \geq \frac{x_{2}}{x_{1}} & (j=1) \\
\left(\frac{x_{2}}{x_{1}}, \frac{1}{x_{1}}-A\right), & \frac{x_{2}}{x_{1}} \geq \frac{1}{x_{1}}-A \quad(j=2)
\end{array}, \quad A:=\left[\frac{1}{x_{1}}\right]\right.
$$

(compare [23], p. 48ff). All matrices of type 0 have been removed, and the new partition is defined by the types $j=1,2$ and the partial quotients $A \in \mathbb{N}$ of the algorithm.

In particular, we denote

$$
\begin{aligned}
& X_{M}(1):=\left\{\left(x_{1}, x_{2}\right) \in X_{B}: j\left(x_{1}, x_{2}\right)=1\right\} \\
& X_{M}(2):=\left\{\left(x_{1}, x_{2}\right) \in X_{B}: j\left(x_{1}, x_{2}\right)=2\right\}
\end{aligned}
$$

Similarly to the above, for $t \geq 1, j^{(t)}:=j\left(T_{M}^{t-1}\left(x_{1}^{(0)}, x_{2}^{(0)}\right)\right)$ and $A^{(t)}:=$ $A\left(T_{M}^{t-1}\left(x_{1}^{(0)}, x_{2}^{(0)}\right)\right)$. The cylinders are defined by the pairs $\left(j^{(t)}, A^{(t)}\right)$, while the inverses $\beta_{M}^{(t)}:=\beta_{M}\left(j^{(t)}, A^{(t)}\right)$, as well as the convergence matrices $\Omega_{M}^{(t)}$, are defined as above. 


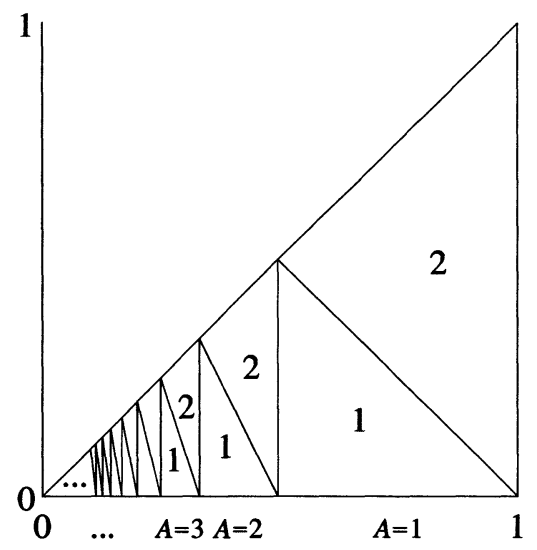

FIGURE 2. The time-1-partition of the multiplicative acceleration of Brun's Algorithm

\section{The natural extension $(\bar{X}, \bar{T})$}

Following [7], we may describe the law of singularization in defining a singularization area i.e., a set $S$ such that every $x \in S$ specifies a matrix $\beta$ to be singularized. To describe $S$ we use the natural extension of a fibred system, introduced by Nakada, Ito and Tanaka [16] (see also [15] and [3]) but we follow F. Schweiger ([23], p. 22f).

Definition. Let $(X, T)$ be a multidimensional continued fraction algorithm. A fibred system $\left(X^{\#}, T^{\#}\right)$ is called a dual algorithm if

(1) $\left(i^{(1)}, \ldots, i^{(t)}\right)$ is an admissible block of digits for $T$ if and only if $\left(i^{(t)}, \ldots, i^{(1)}\right)$ is an admissible block of digits for $T^{\#}$;

(2) there is a partition $X^{\#}(i)$ such that the matrices $\alpha^{\#}(i)$ of $T^{\#}$ restricted to $X^{\#}(i)$ are the transposed matrices of $\alpha(i)$.

Similar to the above, let $V^{\#}(i): T^{\#} X^{\#}(i) \rightarrow X^{\#}(i)$ denote the local inverse branches of $T^{\#}$.

Definition. For any $x \in X$, the polar set $D(x)$ is defined as follows:

$$
D(x):=\left\{y \in X^{\#}: x \in \bigcap_{t=1}^{\infty} T^{t} X\left(i^{(t)}(y), \ldots, i^{(1)}(y)\right)\right\} .
$$

Let $y \in X^{\#}\left(i^{(1)}, \ldots, i^{(t)}\right)$. By the definition, $y \in D(x)$ if and only if $V\left(i^{(t)}, \ldots, i^{(1)}\right)(x)$ is well defined for all $t$. In particular, if all cylinders are full, then $D(x)=X^{\#}$. 
Definition. The dynamical system $(\bar{X}, \bar{T})$, where $\bar{X}:=\{(x, y): x \in X$, $y \in D(x)\}$ and

$$
\bar{T}: \bar{X} \rightarrow \bar{X}, \quad \bar{T}(x, y)=\left(T(x), V^{\#}(i(x))(y)\right)
$$

is called a natural extension of $(X, T)$.

Definition. Let $t \geq 1$. A singularization area is a set $S \subset \bar{X}$ such that, for some fixed $k, \beta^{(t+k)}$ should be singularized if and only if $\left(x^{(t)}, y^{(t)}\right) \in S$.

Remark. In theory, the singularization area could be chosen arbitrarily. However, since the process is based on some matrix identities which have an effect on the remaining matrices, there are some restrictions similar to the ones described in [7] (section 4.2.3). Since we are more 'liberal' in the sense that, throughout this paper, several matrix identities will be used, there is no such general description of these restraints.

In case of Brun's Algorithm, we consider the fibred system $\left(X_{B}, T_{S}\right)$ from above. A dual system can be described as follows. Let

$$
X_{S}^{\#}:=\left\{\left(y_{1}, y_{2}\right): 0 \leq y_{1} ; 0 \leq y_{2} \leq 1\right\}
$$

and set in particular

$$
\begin{aligned}
& X_{S}^{\#}(0):=\left\{\left(y_{1}, y_{2}\right) \in X_{S}^{\#}: 1 \leq y_{1}\right\}, \\
& X_{S}^{\#}(1):=\left\{\left(y_{1}, y_{2}\right): 1 \geq y_{1} \geq y_{2} \geq 0\right\}, \\
& X_{S}^{\#}(2):=\left\{\left(y_{1}, y_{2}\right): 1 \geq y_{2} \geq y_{1} \geq 0\right\} .
\end{aligned}
$$

Define $V_{S}^{\#}: X_{S}^{\#} \rightarrow X_{S}^{\#}$

$$
\begin{gathered}
V_{S}^{\#}(j)\left(y_{1}, y_{2}\right)= \begin{cases}\left(1+y_{1}, y_{2}\right) & (j=0), \\
\left(\frac{1}{1+y_{1}}, \frac{y_{2}}{1+y_{1}}\right) & (j=1), \\
\left(\frac{y_{2}}{1+y_{1}}, \frac{1}{1+y_{1}}\right) & (j=2),\end{cases} \\
\bar{X}_{S}:=X_{B} \times X_{S}^{\#} \text {, and finally } \bar{T}_{S}: \bar{X}_{S} \rightarrow \bar{X}_{S}, \\
\bar{T}_{S}\left(x_{1}, x_{2}, y_{1}, y_{2}\right)=\left(T_{S}\left(x_{1}, x_{2}\right), V_{S}^{\#}\left(j\left(x_{1}, x_{2}\right)\right)\left(y_{1}, y_{2}\right)\right) .
\end{gathered}
$$

Then $\left(\bar{X}_{S}, \bar{T}_{S}\right)$ is a natural extension of $\left(X_{B}, T_{S}\right)$. We now define the singularization area

$$
S_{M}:=X_{B} \times([1, \infty) \times[0,1]),
$$

and thus restate the law of singularization $\mathrm{LM}^{*}$ :

Law of singularization LM: Singularize $\beta_{S}^{(t)}$ if and only if

$$
\left(x_{1}^{(t)}, x_{2}^{(t)}, y_{1}^{(t)}, y_{2}^{(t)}\right) \in S_{M}
$$

using matrix identities $M_{1}$ and $M_{2}$.

By the definition of $S_{M}$, the laws of singularization LM and LM* are equivalent. 

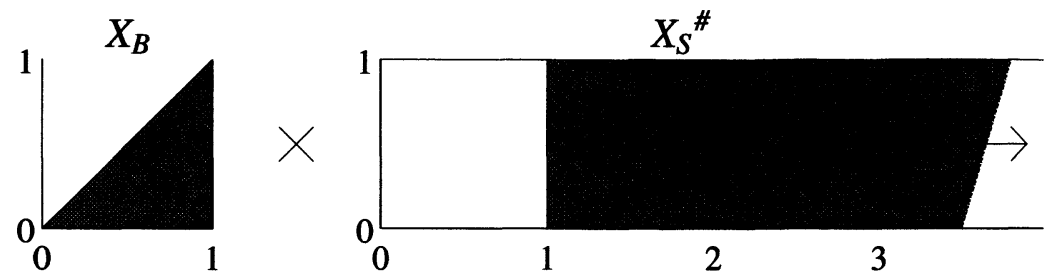

FiguRE 3. The singularization area $S_{M}$

Remark. The matrix identities $M_{1}$ and $M_{2}$, and thus the law of singularization LM, slightly differ from the process of singularization described in [7] and [9] (compare matrix identity $1_{1}$ given in Section 5). Nevertheless, there exists a relation similar to LM between the one-dimensional regular continued fraction algorithm and the Lehner expansions [11]. Lehner expansions are generated by a map isomorphic to Brun's Algorithm $T_{B}:[0,1) \rightarrow[0,1)$,

$$
T_{B}(x)= \begin{cases}\frac{x}{1-x}, & 0 \leq x<\frac{1}{2} ; \\ \frac{1-x}{x}, & \frac{1}{2} \leq x<1 .\end{cases}
$$

This relation, again based on ideas similar to singularization, is described in Dajani and Kraaikamp [5] (see also Ito [8]).

\section{Eliminating partial quotients $A^{(t)}=1$, where $j^{(t)}=1$}

From now on, we assume that the law of singularization LM has already been applied to Brun's Algorithm $T_{S}$ i.e., in the following, we consider the resulting multiplicative acceleration of Brun's Algorithm $T_{M}$.

We are now going to define another singularization process: The Singularization of matrices $\beta_{M}(1,1)$, which will lead to a new algorithm $T_{1}$ with better approximation properties (as opposed to both $T_{S}$ and $T_{M}$ ). We use the following identities:

type $1_{1}$ :

$$
\begin{gathered}
\left(\begin{array}{ccc}
A_{1} & 1 & 0 \\
\epsilon_{1} & 0 & 0 \\
0 & 0 & 1
\end{array}\right)\left(\begin{array}{lll}
1 & 1 & 0 \\
1 & 0 & 0 \\
0 & 0 & 1
\end{array}\right)\left(\begin{array}{ccc}
A_{3} & \phi_{3} & \psi_{3} \\
1 & 0 & 0 \\
0 & \psi_{3} & \phi_{3}
\end{array}\right) \\
=\left(\begin{array}{ccc}
A_{1}+1 & 1 & 0 \\
\epsilon_{1} & 0 & 0 \\
0 & 0 & 1
\end{array}\right)\left(\begin{array}{ccc}
A_{3}+1 & \phi_{3} & \psi_{3} \\
-1 & 0 & 0 \\
0 & \psi_{3} & \phi_{3}
\end{array}\right) .
\end{gathered}
$$

type $1_{2}$ :

$$
\begin{gathered}
\left(\begin{array}{ccc}
A_{1} & 0 & 1 \\
\epsilon_{1} & 0 & 0 \\
0 & 1 & 0
\end{array}\right)\left(\begin{array}{lll}
1 & 1 & 0 \\
1 & 0 & 0 \\
0 & 0 & 1
\end{array}\right)\left(\begin{array}{ccc}
A_{3} & \phi_{3} & \psi_{3} \\
1 & 0 & 0 \\
0 & \psi_{3} & \phi_{3}
\end{array}\right) \\
=\left(\begin{array}{ccc}
A_{1} & 0 & 1 \\
\epsilon_{1} & 0 & 0 \\
1 & 1 & 0
\end{array}\right)\left(\begin{array}{ccc}
A_{3}+1 & \phi_{3} & \psi_{3} \\
-1 & 0 & 0 \\
0 & \psi_{3} & \phi_{3}
\end{array}\right) .
\end{gathered}
$$


Remark. The matrix identity $1_{1}$ corresponds to the identity used in [7] and [9] to define the singularization process for the one-dimensional (regular) continued fraction algorithm.

Define, for $\epsilon \in\{-1,1\}$, the matrices $\beta_{1}(j, A, \epsilon, C)$ as

$$
\beta_{1}(1, A, \epsilon, C)=\left(\begin{array}{ccc}
A & 1 & 0 \\
\epsilon & 0 & 0 \\
C & 0 & 1
\end{array}\right), \quad \beta_{1}(2, A, \epsilon, C)=\left(\begin{array}{ccc}
A & 0 & 1 \\
\epsilon & 0 & 0 \\
C & 1 & 0
\end{array}\right) .
$$

Consider a block of pairs of digits $\left(\left(j^{(t)}, A^{(t)}\right),(1,1),\left(j^{(t+2)}, A^{(t+2)}\right)\right)$. If we singularize $\beta_{M}^{(t+1)}$, then $\beta_{M}^{(t)}$ will be replaced by $\beta_{1}\left(1, A^{(t)}+1,1,0\right)$ (if $j^{(t)}=$ 1 ), or by $\beta_{1}\left(2, A^{(t)}, 1,1\right)$ (if $\left.j^{(t)}=2\right)$. The matrix $\beta_{M}^{(t+2)}$ will be replaced by $\beta_{1}\left(j^{(t+2)}, A^{*(t+2)}+1,-1,0\right)$. Hence, even if $A^{(t)}=1$ and $j^{(t)}=1$, or $A^{(t+2)}=1$ and $j^{(t+2)}=1$, we cannot singularize one of the resulting matrices using the above identities. In other words, from every block of consecutive matrices $\beta_{M}(1,1)$ we can only singularize every other matrix.

We thus have to specify which of the matrices of such blocks should be singularized, and this choice determines the outcome, as it can be seen easily with the following example: Let $\left\{\beta_{M}^{(s)}\right\}_{s=0}^{\infty}$ be a sequence of matrices specified by the pairs of digits $\left(\left(j^{(1)}, A^{(1)}\right), \ldots,\left(j^{(t)}, A^{(t)}\right),(1,1),(1,1),(1,1)\right.$, $\left.\left(j^{(t+4)}, A^{(t+4)}\right)\right)$, where both $A^{(t)} \neq 1$ and $A^{(t+4)} \neq 1$. Singularizing $\beta_{M}^{(t+2)}$ obviously yields a different subsequence of $\left\{\beta_{M}^{(s)}\right\}_{s=0}^{\infty}$, and thus a different algorithm, than singularizing both $\beta_{M}^{(t+1)}$ and $\beta_{M}^{(t+3)}$. The class of S-expansions for the regular one-dimensional continued fraction algorithm was obtained in giving different laws of singularization i.e., different choices of matrices to be singularized, for one single matrix identity. For example, the following law of singularization can be considered:

Law of singularization L1*: From every block of $\boldsymbol{n}$ consecutive matrices $\beta_{M}(1,1)$, singularize the first, the third,... matrices, using identities $1_{1}$ and $1_{2}$.

Remark. Due to the nature of matrix identities $1_{1}$ and $1_{2}$, we may not apply L1* until the first index $s$ with $j^{(s)} \neq 1$ or $A^{(s)} \neq 1$. Strictly speaking, L1* is only valid for matrices $\beta_{M}^{(t)}(1,1)$ where $t>t_{0}$, and $t_{0}:=\min \{s:$ $j^{(s)}=2$ or $\left.A^{(s)}>1\right\}$. Similar restrictions will be true for all laws of singularization proposed from now on.

Using the natural extension $\left(\bar{X}_{M}, \bar{T}_{M}\right)$ of $\left(X_{B}, T_{M}\right)$, where

$$
X_{M}^{\#}:=\left\{\left(y_{1}, y_{2}\right): 0 \leq y_{1} \leq 1 ; 0 \leq y_{2} \leq 1\right\}
$$




$$
\begin{aligned}
X_{M}^{\#}(1) & :=\left\{\left(y_{1}, y_{2}\right) \in X_{M}^{\#}: y_{1} \geq y_{2}\right\} \\
X_{M}^{\#}(2) & :=\left\{\left(y_{1}, y_{2}\right) \in X_{M}^{\#}: y_{2} \geq y_{1}\right\} \\
\bar{X}_{M} & :=X_{B} \times X_{M}^{\#}
\end{aligned}
$$

and $V_{M}^{\#}: X_{M}^{\#} \rightarrow X_{M}^{\#}$

$$
V_{M}^{\#}(j, A)\left(y_{1}, y_{2}\right):= \begin{cases}\left(\frac{1}{A+y_{1}}, \frac{y_{2}}{A+y_{1}}\right) & (j=1), \\ \left(\frac{y_{2}}{A+y_{1}}, \frac{1}{A+y_{1}}\right) & (j=2),\end{cases}
$$

such that $\bar{T}_{M}: \bar{X}_{M} \rightarrow \bar{X}_{M}$ is defined by

$$
\bar{T}_{M}\left(x_{1}, x_{2}, y_{1}, y_{2}\right)=\left(T_{M}\left(x_{1}, x_{2}\right), V_{M}^{\#}\left(j\left(x_{1}, x_{2}\right), A\left(x_{1}, x_{2}\right)\right)\left(y_{1}, y_{2}\right)\right) \text {. }
$$

Again, we may restate $\mathrm{L} 1^{*}$ in terms of a singularization area $S_{1} \subset \bar{X}_{M}$. We use $V_{M}^{\#}$ to control the preceding pairs of digits $\left(j^{(t-1)}, A^{(t-1)}\right),\left(j^{(t-2)}\right.$, $\left.A^{(t-2)}\right), \ldots$ Denote $f_{i}$ the $i^{\text {th }}$ term of Fibonacci's sequence with initial terms $f_{0}=0, f_{1}=1$, and let $\Delta\left(P_{1}, P_{2}, P_{3}\right)$ be the triangle defined by the vertices $P_{1}, P_{2}$ and $P_{3}$. Then

$$
\begin{aligned}
S_{1}:=X_{B}(1) \times\left(\bigcup_{i=0}^{\infty} \Delta\left(\left(\frac{f_{2 i}}{f_{2 i+1}}, 0\right),\left(\frac{f_{2 i}}{f_{2 i+1}}, \frac{1}{f_{2 i+1}}\right),\left(\frac{f_{2 i+1}}{f_{2 i+2}}, \frac{1}{f_{2 i+2}}\right)\right)\right. \\
\left.\cup \bigcup_{i=0}^{\infty} \Delta\left(\left(\frac{f_{2 i}}{f_{2 i+1}}, 0\right),\left(\frac{f_{2 i+2}}{f_{2 i+3}}, 0\right),\left(\frac{f_{2 i+2}}{f_{2 i+3}}, \frac{1}{f_{2 i+3}}\right)\right)\right) .
\end{aligned}
$$

Law of singularization L1: Singularize $\beta_{M}^{(t)}$ if and only if

$$
\left(x_{1}^{(t-1)}, x_{2}^{(t-1)}, y_{1}^{(t-1)}, y_{2}^{(t-1)}\right) \in S_{1}
$$

using identities $1_{1}$ or $1_{2}$, accordingly.
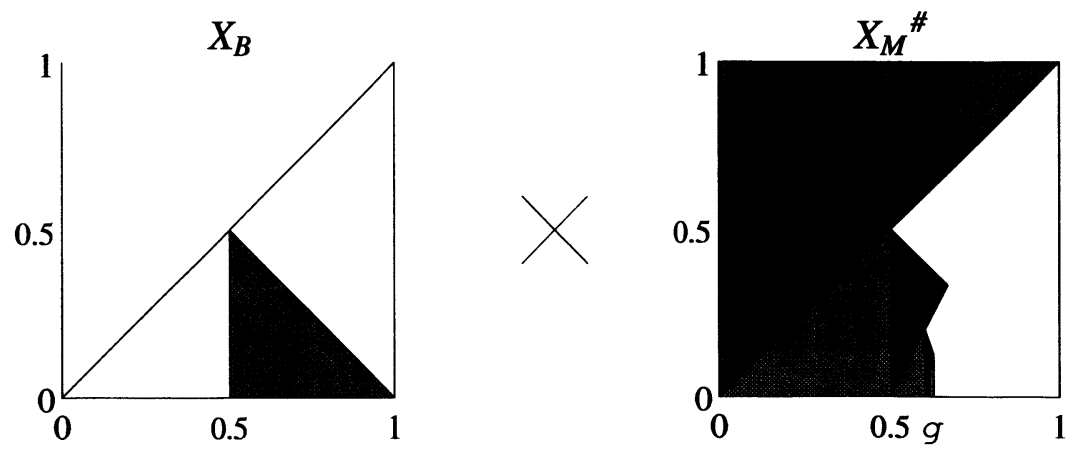

FIGURE 4. The singularization area $S_{1}\left(g=\frac{\sqrt{5}-1}{2}\right)$ 
Thus L1 is equivalent to L1*. The singularization yields a new algorithm, which acts on the following set (compare Figure 5):

$$
\begin{aligned}
X_{1}:=\left\{\left(x_{1}, x_{2}\right)\right. & \left.: 0 \leq x_{2} \leq\left|x_{1}\right| \leq 1 / 2\right\} \\
\cup & \left\{\left(x_{1}, x_{2}\right): 1 / 2 \leq x_{1} \leq 1,1-x_{1} \leq x_{2} \leq x_{1}\right\}
\end{aligned}
$$

or, more precisely,

$$
\begin{aligned}
X_{1}(1):= & \left\{\left(x_{1}, x_{2}\right) \in X_{1}: \frac{x_{2}}{\left|x_{1}\right|} \leq \frac{1}{\left|x_{1}\right|}-A \leq \frac{1}{2}\right\} \\
& \cup\left\{\left(x_{1}, x_{2}\right) \in X_{1}: \begin{array}{l}
\max \left\{\frac{1}{2}, \frac{x_{2}}{\mid x_{1}}\right\} \leq \frac{1}{\left|x_{1}\right|}-A \\
A+1-\frac{1}{\left|x_{1}\right|} \leq \frac{x_{2}}{\left|x_{1}\right|}
\end{array}\right\}, \\
X_{1}(2):= & \left\{\left(x_{1}, x_{2}\right) \in X_{1}: \frac{1}{\left|x_{1}\right|}-A \leq \frac{x_{2}}{\left|x_{1}\right|} \leq \frac{1}{2}\right\} \\
& \cup\left\{\left(x_{1}, x_{2}\right) \in X_{1}: \max \left\{\frac{1}{2}, \frac{1}{\left|x_{1}\right|}-A,(A+1)-\frac{1}{\left|x_{1}\right|}\right\} \leq \frac{x_{2}}{\left|x_{1}\right|}\right\}, \\
X_{1}(3):= & \left\{\left(x_{1}, x_{2}\right) \in X_{1}: \max \left\{\frac{1}{2}, \frac{x_{2}}{\left|x_{1}\right|}\right\} \leq \frac{1}{\left|x_{1}\right|}-A \& \frac{x_{2}}{\left|x_{1}\right|} \leq A+1-\frac{1}{\left|x_{1}\right|}\right\} \\
= & \bigcup_{A=2}^{\infty} \Delta\left(\left(\frac{1}{A+1}, 0\right),\left(\frac{2}{2 A+1}, 0\right),\left(\frac{2}{2 A+1}, \frac{1}{2 A+1}\right)\right) \\
& \cup \bigcup_{A=2}^{\infty} \Delta\left(\left(-\frac{1}{A+1}, 0\right),\left(-\frac{2}{2 A+1}, 0\right),\left(-\frac{2}{2 A+1}, \frac{1}{2 A+1}\right)\right), \\
X_{1}(4):= & \left\{\left(x_{1}, x_{2}\right) \in X_{1}: \max \left\{\frac{1}{2}, \frac{1}{\left|x_{1}\right|}-A\right\} \leq \frac{x_{2}}{\left|x_{1}\right|} \leq A+1-\frac{1}{\left|x_{1}\right|}\right\} \\
= & \bigcup_{A=1}^{\infty} \Delta\left(\left(\frac{1}{A}, \frac{1}{A}\right),\left(\frac{1}{A}, \frac{1}{2 A}\right),\left(\frac{2}{2 A+1}, \frac{1}{2 A+1}\right)\right) \\
& \cup \bigcup_{A=2}^{\infty} \Delta\left(\left(-\frac{1}{A}, \frac{1}{A}\right),\left(-\frac{1}{A}, \frac{1}{2 A}\right),\left(-\frac{2}{2 A+1}, \frac{1}{2 A+1}\right)\right) .
\end{aligned}
$$

The resulting algorithm $T_{1}: X_{1} \rightarrow X_{1}$ (the one defined by the new matrices $\left.\beta_{1}^{(t)}\right)$ can be described as follows:

$$
\begin{gathered}
T_{1}\left(x_{1}, x_{2}\right)= \begin{cases}\left(\frac{1}{\left|x_{1}\right|}-A, \frac{x_{2}}{\left|x_{1}\right|}\right) & (j=1), \\
\left(\frac{x_{2}}{\mid x_{1}}, \frac{1}{\left|x_{1}\right|}-A\right) & (j=2), \\
\left(\frac{1}{\left|x_{1}\right|}-(A+1), \frac{x_{2}}{\left|x_{1}\right|}\right) & (j=3), \\
\left(\frac{x_{2}}{\left|x_{1}\right|}-1, \frac{1}{\left|x_{1}\right|}-A\right) & (j=4),\end{cases} \\
\left(x_{1}, x_{2}\right) \in X_{1}(j), \quad A:=\left[\frac{1}{\left|x_{1}\right|}\right] .
\end{gathered}
$$


We may further define an algorithm $V_{1}^{\#}: X_{1}^{\#} \rightarrow X_{1}^{\#}$, where

$$
\begin{aligned}
& X_{1}^{\#}(1):=\left\{\left(y_{1}, y_{2}\right): 0 \leq y_{2} \leq y_{1} \leq \frac{1}{2}\right\} \\
& X_{1}^{\#}(2):=X_{M}^{\#}(2) \text {, } \\
& X_{1}^{\#}(3):=\left\{\left(y_{1}, y_{2}\right): 0 \leq y_{2} \leq-y_{1} \leq \frac{1}{3}\right\} \\
& \cup \bigcup_{i=1}^{\infty} \Delta\left(\left(-\frac{f_{2 i+2}}{f_{2 i+4}}, 0\right),\left(-\frac{f_{2 i+2}}{f_{2 i+4}}, \frac{1}{f_{2 i+4}}\right),\left(-\frac{f_{2 i}}{f_{2 i+2}}, 0\right)\right) \\
& \cup \bigcup_{i=1}^{\infty} \Delta\left(\left(-\frac{f_{2 i+1}}{f_{2 i+3}}, \frac{1}{f_{2 i+3}}\right),\left(-\frac{f_{2 i}}{f_{2 i+2}}, \frac{1}{f_{2 i+2}}\right),\left(-\frac{f_{2 i}}{f_{2 i+2}}, 0\right)\right), \\
& X_{1}^{\#}(4):=\left\{\left(y_{1}, y_{2}\right): 0 \leq-y_{1} \leq \min \left\{y_{2}, 1-y_{2}\right\} \& y_{2} \leq 1\right\} \\
& X_{1}^{\#}:=\bigcup_{i=1}^{4} X_{1}^{\#}(i) \\
& V_{1}^{\#}(j, A)\left(y_{1}, y_{2}\right)= \begin{cases}\left(\frac{1}{A+y_{1}}, \frac{y_{2}}{A+y_{1}}\right) & (j=1), \\
\left(\frac{y_{2}}{A+y_{1}}, \frac{1}{A+y_{1}}\right) & (j=2), \\
\left(-\frac{y_{2}}{A+1+y_{1}}, \frac{y_{2}}{A+1+y_{1}}\right) & (j=3), \\
\left(-\frac{y_{2}}{A+y_{1}+y_{2}}, \frac{1}{A+y_{1}+y_{2}}\right) & (j=4) .\end{cases}
\end{aligned}
$$

Since the cylinders of the new algorithm are not full, we verify that

$$
D_{1}\left(x_{1}, x_{2}\right)= \begin{cases}X_{1}^{\#}(1) \cup X_{1}^{\#}(2) & \text { if } 0 \leq x_{1}, \\ X_{1}^{\#}(3) \cup X_{1}^{\#}(4) & \text { if } x_{1} \leq 0\end{cases}
$$

i.e., whenever $x_{1} \leq 0$, so is $y_{1}$ (and conversely). Thus $D_{1}\left(x_{1}, x_{2}\right)$ is not empty. Finally, we put (see Figure 5):

$$
\begin{aligned}
\bar{X}_{1}:= & \left\{\left(x_{1}, x_{2}\right) \in X_{1}: 0 \leq x_{1}\right\} \times\left(X_{1}^{\#}(1) \cup X_{1}^{\#}(2)\right) \\
& \cup\left\{\left(x_{1}, x_{2}\right) \in X_{1}: x_{1} \leq 0\right\} \times\left(X_{1}^{\#}(3) \cup X_{1}^{\#}(4)\right)
\end{aligned}
$$

and

$$
\bar{T}_{1}\left(x_{1}, x_{2}, y_{1}, y_{2}\right)=\left(T_{1}\left(x_{1}, x_{2}\right), V_{1}^{\#}\left(j\left(x_{1}, x_{2}\right), A\left(x_{1}, x_{2}\right)\right)\left(y_{1}, y_{2}\right)\right)
$$

to obtain the system $\left(\bar{X}_{1}, \bar{T}_{1}\right)$.

\section{The ergodic system connected with the natural extension}

Consider the fibred system $\left(X_{B}, T_{M}\right)$ and its natural extension $\left(\bar{X}_{M}\right.$, $\left.\bar{T}_{M}\right)$. Let $\Sigma_{M}$ be the $\sigma$-algebra generated by the cylinders of $\bar{X}_{M}$. The multiplicative acceleration of Brun's Algorithm is known to be ergodic and 

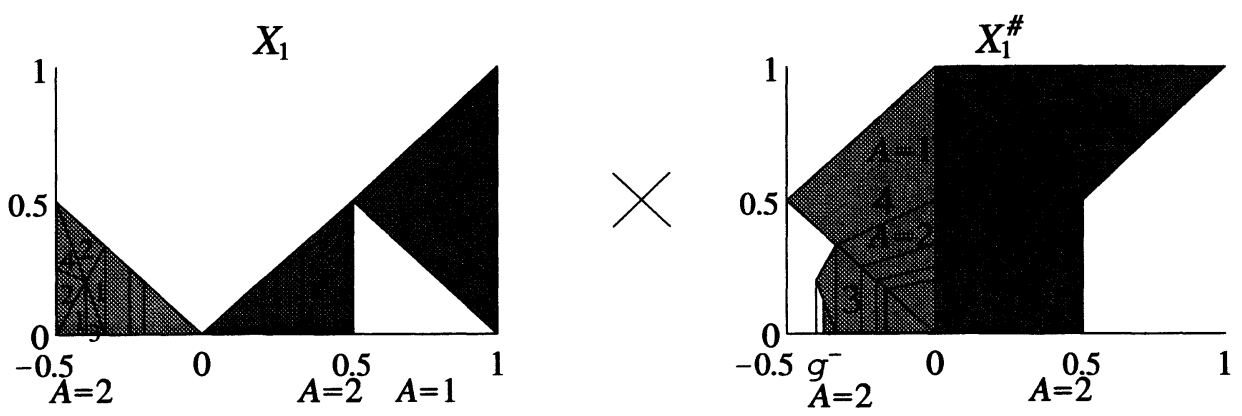

Figure 5. The set $\bar{X}_{1}\left(g^{-}=g-1\right)$

conservative, and it admits an invariant probability measure $\mu_{M}$, whose density is given as

$$
\frac{1}{C_{M}} \frac{1}{\left(1+x_{1} y_{1}+x_{2} y_{2}\right)^{3}}
$$

(see e.g. Schweiger [20] or Arnoux and Nogueira [1]), where $C_{M} \approx 0,19$. Define

$$
\begin{aligned}
& S_{1}^{C}:=\bar{X}_{M} \backslash S_{1}, \\
& S_{1}^{+}:=S_{1}^{C} \backslash \bar{T}_{M} S_{1}, \\
& N_{1}:=\bar{T}_{1} \bar{T}_{M}^{-1} S_{1} .
\end{aligned}
$$

Note that, by the definitions $N_{1} \cap \bar{X}_{M}=\emptyset$ and $\bar{X}_{1}=S_{1}^{+} \cup N_{1}$. Next we define a transformation $\varsigma_{1}$ that 'jumps' over the singularization area $S_{1}$.

Definition. The transformation $\varsigma_{1}: S_{1}^{C} \rightarrow S_{1}^{C}$ is defined by

$$
\varsigma_{1}\left(x_{1}, x_{2}, y_{1}, y_{2}\right)= \begin{cases}\bar{T}_{M}\left(x_{1}, x_{2}, y_{1}, y_{2}\right), & \left(x_{1}, x_{2}, y_{1}, y_{2}\right) \in S_{1}^{C} \backslash \bar{T}_{M}^{-1} S_{1}, \\ \bar{T}_{M}^{2}\left(x_{1}, x_{2}, y_{1}, y_{2}\right), & \left(x_{1}, x_{2}, y_{1}, y_{2}\right) \in \bar{T}_{M}^{-1} S_{1} .\end{cases}
$$

Using the theory of jump transformations (see e.g. [22]), this yields an ergodic system $\left(S_{1}^{C}, \Sigma_{S_{1}^{C}}, \mu_{S_{1}^{C}}, \varsigma_{1}\right)$, where $\Sigma_{S_{1}^{C}}$ is the restriction of $\Sigma_{M}$ to $S_{1}^{C}$ and $\mu_{S_{1}^{C}}$ is the probability induced by $\mu_{M}$ on $\Sigma_{S_{1}^{C}}$. Notice that $C_{S_{1}^{C}}:=$ $\mu_{M}\left(S_{1}^{C}\right) \approx 0,78$. Now we may identify the set $N_{1}$ with $\bar{T}_{M} S_{1}$ by a bijective map $M_{1}: S_{1}^{C} \rightarrow \bar{X}_{1}$, where $M_{1} \bar{T}_{M} S_{1}=N_{1}$, while $M_{1}$ is the identity on $S_{1}^{+}$:

Definition. The map $M_{1}: S_{1}^{C} \rightarrow \bar{X}_{1}$ is defined by,

$$
M_{1}\left(x_{1}, x_{2}, y_{1}, y_{2}\right)= \begin{cases}\left(x_{1}, x_{2}, y_{1}, y_{2}\right), & \left(x_{1}, x_{2}, y_{1}, y_{2}\right) \in S_{1}^{+}, \\ \left(-\frac{x_{1}}{1+x_{1}}, \frac{x_{2}}{1+x_{1}}, y_{1}-1, y_{2}\right), & \left(x_{1}, x_{2}, y_{1}, y_{2}\right) \in \bar{T}_{M} S_{1}\end{cases}
$$


We may illustrate the relations between $S_{1}, \bar{T}_{M} S_{1}$ and $M_{1} \bar{T}_{M} S_{1}$ with two sets $E_{1} \in S_{1}, E_{2} \in S_{1}$, where $E_{1}$ is defined by the block of pairs of digits $((1,2),(1,1),(1,2))\left(i . e ., E_{1}=\left\{\left(x_{1}, x_{2}, y_{1}, y_{2}\right):\left(j^{(0)}, A^{(0)}\right)=\right.\right.$ $\left.\left.(1,2),\left(j^{(1)}, A^{(1)}\right)=(1,1),\left(j^{(2)}, A^{(2)}\right)=(1,2)\right\}\right)$, while $E_{2}$ is defined by $((2$, $2),(1,1),(2,2))$.
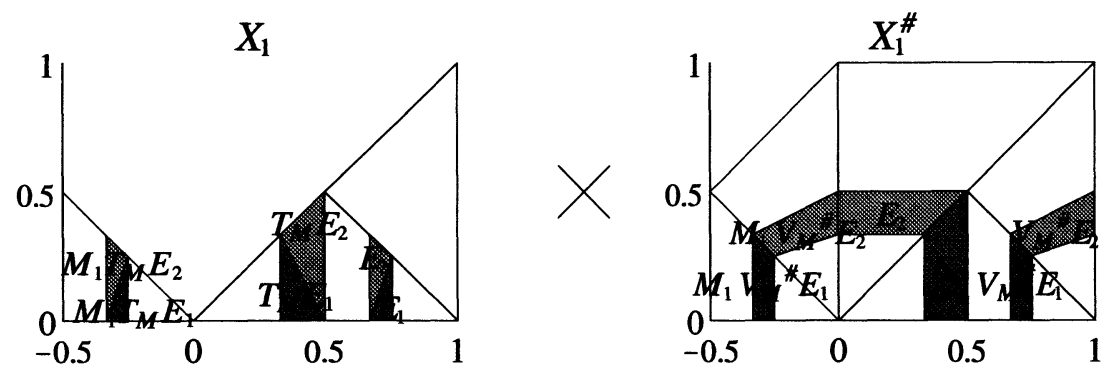

Figure 6. Evolution of the sets $E_{1} \subset S_{1}$ and $E_{2} \subset S_{1}$

We get the following

Theorem 6.1. Consider $\tau_{1}: \bar{X}_{1} \rightarrow \bar{X}_{1}, \tau_{1}\left(x_{1}, x_{2}, y_{1}, y_{2}\right)=M_{1} \varsigma_{1} M_{1}^{-1}$. Then $\left(\bar{X}_{1}, \Sigma_{1}, \mu_{1}, \tau_{1}\right)$ is an ergodic dynamical system, where $\Sigma_{1}$ is the $\sigma$ algebra generated by the cylinders of $\bar{X}_{1}, \mu_{1}$ is the probability measure with density function

$$
\frac{1}{C_{1}} \frac{1}{\left(1+\left|x_{1}\right| y_{1}+x_{2} y_{2}\right)^{3}},
$$

$C_{1}=C_{M} C_{S_{1}^{C}} \approx 0,15$, and for all $\left(x_{1}, x_{2}, y_{1}, y_{2}\right) \in \bar{X}_{1}$,

$$
\tau_{1}\left(x_{1}, x_{2}, y_{1}, y_{2}\right)=\bar{T}_{1}\left(x_{1}, x_{2}, y_{1}, y_{2}\right)
$$

\section{A cyclic version of the algorithm}

Consider the multiplicative acceleration of Brun's Algorithm $T_{M}$ as described above and, for $t$ large enough, the convergence matrix

$$
\Omega_{M}^{(t)}=\left(\begin{array}{lll}
q^{(t)} & q^{\left(t^{\prime}\right)} & q^{\left(t^{\prime \prime}\right)} \\
p_{1}^{(t)} & p_{1}^{\left(t^{\prime}\right)} & p_{1}^{\left(t^{\prime \prime}\right)} \\
p_{2}^{(t)} & p_{2}^{\left(t^{\prime}\right)} & p_{2}^{\left(t^{\prime \prime}\right)}
\end{array}\right)
$$

i.e., the approximations $\left(p_{1}^{(t)} / q^{(t)}, p_{2}^{(t)} / q^{(t)}\right), \quad\left(p_{1}^{\left(t^{\prime}\right)} / q^{\left(t^{\prime}\right)}, p_{2}^{\left(t^{\prime}\right)} / q^{\left(t^{\prime}\right)}\right)$ and $\left(p_{1}^{\left(t^{\prime \prime}\right)} / q^{\left(t^{\prime \prime}\right)}, p_{2}^{\left(t^{\prime \prime}\right)} / q^{\left(t^{\prime \prime}\right)}\right)$ to some $\left(x_{1}^{(0)}, x_{2}^{(0)}\right) \in X_{B}$ generated by the algorithm. Define $P_{M}^{(s)}:=\left(p_{1}^{(s)} / q^{(s)}, p_{2}^{(s)} / q^{(s)}\right)$, then

$$
\left(x_{1}^{(0)}, x_{2}^{(0)}\right) \in \Delta\left(P_{M}^{(t)}, P_{M}^{\left(t^{\prime}\right)}, P_{M}^{\left(t^{\prime \prime}\right)}\right) .
$$




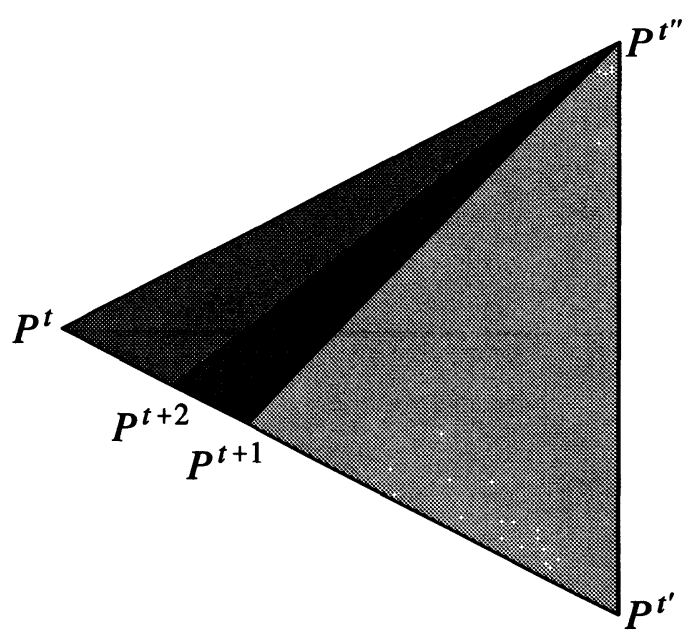

FIGURE 7. Example for an approximation where $j^{(t+1)}=1$

Let $\Gamma\left(P_{1}, P_{2}\right)$ be defined as the line segment between the points $P_{1}$ and $P_{2}$. We observe that, by construction of the approximations, $P_{M}^{(t+1)} \in$ $\Gamma\left(P_{M}^{(t)}, P_{M}^{\left(t^{\prime}\right)}\right)$. Further, as long as $j^{(t+1)}=1, \ldots, j^{(t+i)}=1$ for some $i \geq$ 1 , then $P_{M}^{(t+2)}, \ldots, P_{M}^{(t+i+1)}$ lie on that same line segment. In particular, $P_{M}^{(t+i+1)} \in \Gamma\left(P_{M}^{(t+i)}, P_{M}^{(t+i-1)}\right) \subset \Gamma\left(P_{M}^{(t)}, P_{M}^{\left(t^{\prime}\right)}\right)$. Thus the approximation triangles $\Delta\left(P_{M}^{(t+i+1)}, P_{M}^{\left((t+i+1)^{\prime}\right)}, P_{M}^{\left((t+i+1)^{\prime \prime}\right)}\right)$ get very 'long' i.e., the vertex $P_{M}^{\left(t^{\prime \prime}\right)}$ is not replaced until some $j^{(t+l)}=2, l>i$ (Fig. 7).

On the other hand, if $j^{(t+1)}=2$, then $P_{M}^{(t+2)} \in \Gamma\left(P_{M}^{(t+1)}, P_{M}^{\left(t^{\prime \prime}\right)}\right)$, and both $P_{M}^{\left(t^{\prime}\right)}$ and $P_{M}^{\left(t^{\prime \prime}\right)}$ have been replaced with $P_{M}^{(t+1)}$ and $P_{M}^{(t+2)}$, respectively. We call this a cyclic approximation.

We are now going to construct an algorithm that 'jumps' over the 'bad' (in the above sense) types $j=1$. The following matrix identity (and thus the corresponding law of singularization) somewhat is a generalization of the identity type $1_{2}$ :

type $Q$ :

$$
\begin{gathered}
\left(\begin{array}{ccc}
A_{1} & 0 & 1 \\
B_{1} & 0 & 0 \\
C_{1} & 1 & 0
\end{array}\right)\left(\begin{array}{ccc}
A_{2} & 1 & 0 \\
B_{2} & 0 & 0 \\
0 & 0 & 1
\end{array}\right)\left(\begin{array}{ccc}
A_{3} & \phi_{3} & \psi_{3} \\
1 & 0 & 0 \\
0 & \psi_{3} & \phi_{3}
\end{array}\right) \\
=\left(\begin{array}{ccc}
A_{1} A_{2} & 0 & 1 \\
B_{1} A_{2} & 0 & 0 \\
B_{1}+C_{1} A_{2} & 1 & 0
\end{array}\right)\left(\begin{array}{ccc}
A_{3}+\frac{1}{A_{2}} & \phi_{3} & \psi_{3} \\
-\frac{B_{2}}{A_{2}} & 0 & 0 \\
0 & \psi_{3} & \phi_{3}
\end{array}\right) .
\end{gathered}
$$


Similar to the above, we further generalize the definition of the matrices $\beta_{M}(j, A)(j \in\{1,2\})$ to

$$
\beta(1, A, B, C)=\left(\begin{array}{lll}
A & 1 & 0 \\
B & 0 & 0 \\
C & 0 & 1
\end{array}\right), \quad \beta(2, A, B, C)=\left(\begin{array}{lll}
A & 0 & 1 \\
B & 0 & 0 \\
C & 1 & 0
\end{array}\right),
$$

where $\beta_{M}(j, A)=\beta(j, A, 1,0)$, and define a law of singularization as follows:

Law of singularization LQ*: From any block of matrices $\left(\beta^{(t)}, \ldots, \beta^{(t+i)}\right)$, where $j^{(t)}=1, \ldots, j^{(t+i)}=1$, and both $j^{(t-1)}=2$ and $j^{(t+i+1)}=2$, singularize the first, the second, ... the last matrix, using identity type $Q$.

Or equivalently, in terms of the singularization area $S_{Q}:=X_{M}(1) \times X_{M}^{\#}$,

Law of singularization LQ: Singularize $\beta^{(t)}$ if and only if

$$
\left(x_{1}^{(t-1)}, x_{2}^{(t-1)}, y_{1}^{(t-1)}, y_{2}^{(t-1)}\right) \in S_{Q}
$$

using matrix identity type $Q$.

Similar to LM and $\mathrm{LM}^{*}$, the order of singularizing matrices in $\mathrm{LQ}^{*}$ only is of a certain technical importance, and we could define matrix identities which would allow singularization independent of the order. The resulting algorithm, a 'cyclic' acceleration of Brun's Algorithm, would be the same. Therefore $\mathrm{LQ}$, where the order is not determined, is equivalent to $\mathrm{LQ}^{*}$.

Let $\left[A_{1}, A_{2}, \ldots, A_{s}\right]$ denote the regular one-dimensional continued fraction expansion with partial quotients $A_{1}, A_{2}, \ldots, A_{s}$ i.e.,

$$
\left[A_{1}, A_{2}, \ldots, A_{s}\right]=\frac{1}{A_{1}+\frac{1}{A_{2}+\frac{1}{\ddots \quad+\frac{1}{A_{s}}}}} .
$$

Consider some $t>0$ with $j^{(t)}=2$. Let $k \geq 0, i \geq 0$ be such that $j^{(t-1)}=$ $1, \ldots, j^{(t-k)}=1, j^{(t-k-1)}=2$, and $j^{(t+1)}=1, \ldots, j^{(t+i)}=1, j^{(t+i+1)}=2$. The integers $A^{(t-k-1)}, \ldots, A^{(t)}, \ldots, A^{(t+i+1)}$ are the corresponding partial quotients obtained by the multiplicative acceleration of Brun's Algorithm (the original algorithm).

Further, let the corresponding $t^{*} \leq t$ be such that $P_{Q}^{\left(t^{*}\right)}$ is the $t^{* \text { th }}$ convergent obtained by the new algorithm, where $P_{Q}^{\left(t^{*}\right)}=P_{M}^{(t)}$. By induction, we get the inverse matrices of the resulting algorithm

$$
\beta_{Q}^{\left(t^{*}\right)}\left(x_{1}^{(0)}, x_{2}^{(0)}\right)=\left(\begin{array}{ccc}
A_{1}^{\left(t^{*}\right)} A_{R}^{\left(t^{*}\right)} & 0 & 1 \\
\frac{(-1)^{i}}{\left.A_{R}^{(t)}-1\right)} A_{R}^{\left(t^{*}\right)} & 0 & 0 \\
C^{\left(t^{*}\right)} & 1 & 0
\end{array}\right)
$$


where

$$
\begin{aligned}
& A_{L}^{\left(t^{*}\right)}=A_{L}^{\left(t^{*}\right)}\left(x_{1}^{(0)}, x_{2}^{(0)}\right)=\left[A^{(t)}, \ldots, A^{(t-k)}\right], \\
& A_{R}^{\left(t^{*}\right)}=A_{R}^{\left(t^{*}\right)}\left(x_{1}^{(0)}, x_{2}^{(0)}\right)= \begin{cases}1 & \text { if } i=0, \\
{\left[A^{(t+i)}, \ldots, A^{(t+1)}\right] \cdots\left[A^{(t+1)}\right]} & \text { if } i>0,\end{cases} \\
& C^{\left(t^{*}\right)}=C^{\left(t^{*}\right)}\left(x_{1}^{(0)}, x_{2}^{(0)}\right)= \begin{cases}0 & \text { if } i=0, \\
1 & \text { if } i=1, \\
{\left[A^{(t+i)}, \ldots, A^{(t+2)}\right] \cdots\left[A^{(t+2)}\right]} & \text { if } i>1 .\end{cases}
\end{aligned}
$$

Now let $i \geq 0, A_{1} \geq 1, \ldots, A_{i+1} \geq 1$ and define

$$
\begin{aligned}
E_{1}= & E_{1}\left(i ; A_{1}, \ldots, A_{i}, A_{i+1}\right):=\left(\frac{(-1)^{i}}{\left[A_{i+1}, \ldots, A_{1}\right] \cdots\left[A_{1}\right]}, 0\right) \\
E_{2}= & E_{2}\left(i ; A_{1}, \ldots, A_{i}, A_{i+1}\right):=\left(\frac{(-1)^{i}}{\left[A_{i+1}, \ldots, A_{1}\right] \cdots\left[A_{1}\right]}, \frac{1}{\left[A_{i+1}, \ldots, A_{1}\right]}\right) \\
E_{3}= & E_{3}\left(i ; A_{1}, \ldots, A_{i}, A_{i+1}\right):= \\
& \left(\frac{(-1)^{i}}{\left[A_{i+1}+1, A_{i}, \ldots, A_{1}\right] \cdot\left[A_{i}, \ldots, A_{1}\right] \cdots\left[A_{1}\right]}, \frac{1}{\left[A_{i+1}+1, A_{i}, \ldots, A_{1}\right]}\right) .
\end{aligned}
$$

Then

$$
X_{Q}=\bigcup_{i=1}^{\infty} \bigcup_{A_{1}=1}^{\infty} \cdots \bigcup_{A_{i+1}=1}^{\infty} \Delta\left(E_{1}, E_{2}, E_{3}\right) \subset[-1,1] \times[0,1]
$$

Remark. As above, $i$ is the length of a block of consecutive matrices of type $j=1$, and $A_{1}, \ldots, A_{i}$ are the corresponding partial quotients (resulting from the original algorithm). $A_{i+1}$ is the partial quotient corresponding to the first type $j_{i+1}=2$. If $i=0$, then $\Delta\left(E_{1}, E_{2}, E_{3}\right)$ reduces to $\Delta\left(\left(\frac{1}{A_{1}}, 0\right),\left(\frac{1}{A_{1}}, \frac{1}{A_{1}}\right),\left(\frac{1}{A_{1}+1}, \frac{1}{A_{1}+1}\right)\right)$, and

$$
\bigcup_{A_{1}=1}^{\infty} \Delta\left(\left(\frac{1}{A_{1}}, 0\right),\left(\frac{1}{A_{1}}, \frac{1}{A_{1}}\right),\left(\frac{1}{A_{1}+1}, \frac{1}{A_{1}+1}\right)\right)=X_{M}(2)
$$

In principle, the resulting algorithm is defined by the inverse matrices $\beta_{Q}^{(t)}$. However, the actual construction yields the difficulty that the definitions of $A_{L}^{\left(t^{*}\right)}$ and $A_{R}^{\left(t^{*}-1\right)}$ depend on the explicit knowledge of the partial quotients $A^{(t-1)}, \ldots, A^{(t-k)}$. We may overcome this problem in using the 
natural extension: For $i \geq 1$, define

$$
\begin{aligned}
& E_{1}^{\#}= E_{1}^{\#}\left(i ; A_{1}, \ldots, A_{i}\right):=\left(\frac{1}{\left[A_{i}, \ldots, A_{1}\right]}, 0\right), \\
& E_{2}^{\#}=E_{2}^{\#}\left(i ; A_{1}, \ldots, A_{i}\right):=\left(\frac{1}{\left[A_{i}, \ldots, A_{1}\right]}, \frac{1}{\left[A_{i}, \ldots, A_{1}\right] \cdots\left[A_{1}+1\right]}\right), \\
& E_{3}^{\#}=E_{3}^{\#}\left(i ; A_{1}, \ldots, A_{i}\right):= \\
&\left(\frac{1}{\left[A_{i}, \ldots, A_{2}, A_{1}+1\right]}, \frac{1}{\left[A_{i}, \ldots, A_{2}, A_{1}+1\right] \cdots\left[A_{1}+1\right]}\right) .
\end{aligned}
$$

If $i=0$, then $E_{1}^{\#}=(0,0), E_{2}^{\#}=(0,1)$ and $E_{3}^{\#}=(1,1)$. Let

$$
\bar{X}_{Q}=\bigcup_{i=0}^{\infty} \bigcup_{A_{1}=1}^{\infty} \cdots \bigcup_{A_{i+1}=1}^{\infty} \Delta\left(E_{1}, E_{2}, E_{3}\right) \times \Delta\left(E_{1}^{\#}, E_{2}^{\#}, E_{3}^{\#}\right) .
$$

We may define a cyclic acceleration of Brun's Algorithm $\bar{T}_{Q}: \bar{X}_{Q} \rightarrow \bar{X}_{Q}$,

$$
\bar{T}_{Q}\left(x_{1}, x_{2}, y_{1}, y_{2}\right)=\left(\frac{A_{R}^{+}}{A_{R}^{-}\left|x_{1}\right|}-A_{L} A_{R}^{+}, \frac{A_{R}^{+} x_{2}}{A_{R}^{-}\left|x_{1}\right|}-C, V_{M}^{\# k^{++1}}\left(y_{1}, y_{2}\right)\right)
$$

where

$$
\begin{aligned}
& k^{-}:=\min \left\{t: V_{M}^{t+1} \in X_{M}(2)\right\} \\
& k^{+}:=\min \left\{t: T_{M}^{t+1}\left(\frac{A_{L}^{*} A_{R}^{-}\left|x_{1}\right|}{A_{L}^{*}-A_{R}^{-}\left|x_{1}\right|}, \frac{A_{L}^{*} x_{2}}{A_{L}^{*}-A_{R}^{-}\left|x_{1}\right|}\right) \in X_{M}(2)\right\}, \\
& A_{L}:=\left[A_{1}, \ldots, A_{1-k^{-}}\right] \text {, } \\
& A_{L}^{*}:=\left[A_{L}\right]-A_{L}, \\
& A_{R}^{+}:= \begin{cases}1 & \text { if } k^{+}=0, \\
{\left[A_{k^{+}+1}, \ldots, A_{2}\right] \cdots\left[A_{2}\right]} & \text { if } k^{+}>0 .\end{cases} \\
& A_{R}^{-}:= \begin{cases}1 & \text { if } k^{-}=0 \\
{\left[A_{1}, \ldots, A_{2-k^{-}}\right] \cdots\left[A_{2-k^{-}}\right]} & \text {if } k^{-}>0\end{cases} \\
& C:= \begin{cases}0 & \text { if } k^{+}=0 \\
1 & \text { if } k^{+}=1 . \\
{\left[A_{k^{+}+1}, \ldots, A_{3}\right] \cdots\left[A_{3}\right]} & \text { if } k^{+}>1 .\end{cases} \\
& A_{i}:= \begin{cases}A\left(T_{M}^{i-1}\left(\frac{A_{L}^{*} A_{R}^{-}\left|x_{1}\right|}{A_{L}^{*}-A_{R}^{-}\left|x_{1}\right|}, \frac{A_{L}^{*} x_{2}}{A_{L}^{*}-A_{R}^{-}\left|x_{1}\right|}\right)\right) & \text { if } k^{+} \geq i \geq 1, \\
{\left[\frac{1}{V_{M}^{\#^{i}\left(y_{1}, y_{2}\right)}}\right]} & \text { if } 0 \geq i \geq-k^{-} .\end{cases}
\end{aligned}
$$


Finally, we define

$$
\begin{aligned}
& k^{(t)}:=k^{+}\left(T_{Q}^{t-1}\left(x_{1}^{(0)}, x_{2}^{(0)}\right)\right)=k^{-}\left(T_{Q}^{t}\left(x_{1}^{(0)}, x_{2}^{(0)}\right)\right), \\
& A_{L}^{(t)}:=A_{L}\left(T_{Q}^{t-1}\left(x_{1}^{(0)}, x_{2}^{(0)}\right)\right) \\
& A_{R}^{(t)}:=A_{R}^{+}\left(T_{Q}^{t-1}\left(x_{1}^{(0)}, x_{2}^{(0)}\right)\right)=A_{R}^{-}\left(T_{Q}^{t}\left(x_{1}^{(0)}, x_{2}^{(0)}\right)\right),
\end{aligned}
$$

and

$$
C^{(t)}:=C\left(T_{Q}^{t-1}\left(x_{1}^{(0)}, x_{2}^{(0)}\right)\right)
$$

\section{Convergence properties}

In constructing the cyclic acceleration of the algorithm, we avoid more than three partial convergents lying on a line. Alas, the method yields another problem: While for $k^{(t)}$ even, $\left(x_{1}^{(0)}, x_{2}^{(0)}\right) \in \Delta\left(P_{Q}^{(t)}, P_{Q}^{(t-1)}, P_{Q}^{(t-2)}\right)$, this is not true if $k^{(t)}$ is odd (Fig. 8). To overcome this problem, we have

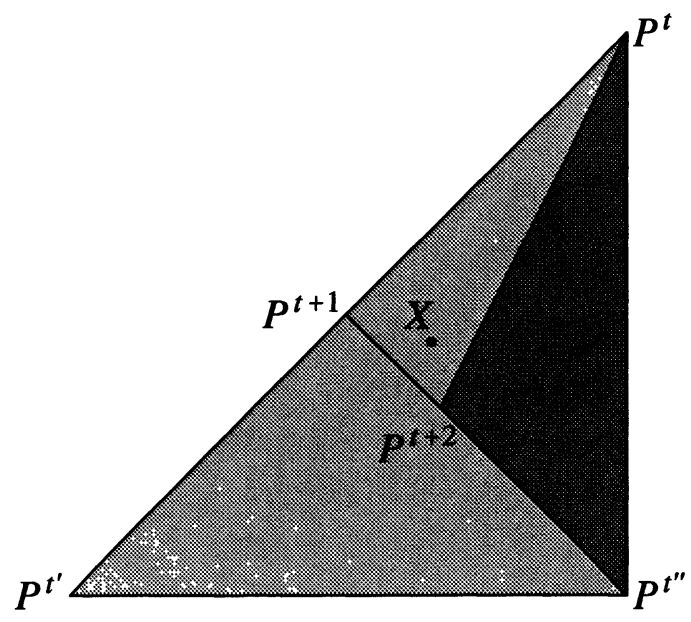

FIGURE 8. Example for an approximation after singularization, where $j^{(t+1)}=2$ and $j^{(t+2)}=1$

to accept single matrices of type $j=1$. We propose the following law of singularization:

Law of singularization Lq*: Let $\left(\beta^{(t)}, \ldots, \beta^{(t+i)}\right)$ be a block of matrices such that $j^{(t)}=1, \ldots, j^{(t+i)}=1$, and both $j^{(t-1)}=2$ and $j^{(t+i+1)}=2$. If $i$ is even, then singularize the first, the second,... the last matrix, using identity type $Q$. If $i$ is odd, and $i \geq 3$, then singularize the first, the 
second,... matrix until (including) the first matrix before the last, using identity type $Q$.

Denote

$$
\begin{aligned}
& F_{1}=F_{1}\left(A_{1}, A_{2}\right):=\left(\frac{1}{A_{2}+\frac{1}{A_{1}}}, 0\right), \\
& F_{2}=F_{2}\left(A_{1}, A_{2}\right):=\left(\frac{1}{A_{2}+\frac{1}{A_{1}+1}}, 0\right), \\
& F_{3}=F_{3}\left(A_{1}, A_{2}\right):=\left(\frac{1}{A_{2}+\frac{1}{A_{1}}}, \frac{1}{A_{1}\left(A_{2}+\frac{1}{A_{1}}\right)}\right) \\
& F_{4}=F_{4}\left(A_{1}, A_{2}\right):=\left(\frac{1}{A_{2}+\frac{1}{A_{1}+1}}, \frac{1}{\left(A_{1}+1\right)\left(A_{2}+\frac{1}{A_{1}+1}\right)}\right) .
\end{aligned}
$$

Setting

$$
\begin{aligned}
S_{q}:= & \bigcup_{A_{1}=1}^{\infty} \bigcup_{A_{2}=1}^{\infty} \Delta\left(F_{1}, F_{2}, F_{3}\right) \times X_{M}^{\#} \\
& \cup \bigcup_{A_{1}=1}^{\infty} \bigcup_{A_{2}=1}^{\infty} \Delta\left(F_{1}, F_{3}, F_{4}\right) \times \bigcup_{i=0}^{\infty} \bigcup_{A_{1}=1}^{\infty} \cdots \bigcup_{A_{2 i+2}=1}^{\infty} \Delta\left(E_{1}^{\#}, E_{2}^{\#}, E_{3}^{\#}\right),
\end{aligned}
$$

we find $\mathrm{Lq}$ as an equivalent law of singularization:

Law of singularization Lq: Singularize $\beta^{(t)}$ if and only if

$$
\left(x_{1}^{(t-1)}, x_{2}^{(t-1)}, y_{1}^{(t-1)}, y_{2}^{(t-1)}\right) \in S_{q},
$$

using matrix identity type $Q$.

Define

$$
\begin{aligned}
E_{4}= & E_{4}\left(i ; A_{1}, \ldots, A_{i}, A_{i+1}\right) \\
:= & \left(\frac{(-1)^{i}}{\left[A_{i+1}+1, A_{i}, \ldots, A_{1}\right] \cdot\left[A_{i}, \ldots, A_{1}\right] \cdots\left[A_{1}\right]}, 0\right), \\
\bar{X}_{q}(1): & \bigcup_{i=0}^{\infty} \bigcup_{A_{1}=1}^{\infty} \cdots \bigcup_{A_{2 i+1}=1}^{\infty} \Delta\left(E_{1}, E_{2}, E_{3}\right) \times \Delta\left(E_{1}^{\#}, E_{2}^{\#}, E_{3}^{\#}\right), \\
\bar{X}_{q}(2):= & \bigcup_{i=1}^{\infty} \bigcup_{A_{1}=1}^{\infty}\left(\Delta\left(E_{1}, E_{2}, E_{3}\right) \times \bigcup_{A_{1}=2}^{\infty} \cdots \bigcup_{A_{2 i+2}=1}^{\infty}(1) \Delta\left(E_{1}^{\#}, E_{2}^{\#}, E_{3}^{\#}\right)\right) \\
& \cup \bigcup_{i=1}^{\infty} \bigcup_{A_{1}=0}^{\infty} \cdots \bigcup_{A_{2 i+1}=1}^{\infty} \Delta\left(E_{1}, E_{3}, E_{4}\right) \times \Delta\left(E_{1}^{\#}, E_{2}^{\#}, E_{3}^{\#}\right),
\end{aligned}
$$

and consequently,

$$
\bar{X}_{q}:=\bar{X}_{q}(1) \cup \bar{X}_{q}(2)
$$


The resulting algorithm $\bar{T}_{q}: \bar{X}_{q} \rightarrow \bar{X}_{q}$ can be defined similarly as above:

$$
\begin{aligned}
& \bar{T}_{q}\left(x_{1}, x_{2}, y_{1}, y_{2}\right)= \\
& \begin{cases}\left(\frac{x_{2}}{A_{R}^{-}\left|x_{1}\right|}, \frac{1}{A_{R}^{-}\left|x_{1}\right|}-A_{L}, V_{M}^{\#}\left(y_{1}, y_{2}\right)\right) & \text { if } j=1, \\
\left(\frac{A_{R}^{+}}{A_{R}^{-}\left|x_{1}\right|}-A_{L} A_{R}^{+}, \frac{A_{R}^{+} x_{2}}{A_{R}^{-}\left|x_{1}\right|}-C, V_{M}^{\# k^{+}+1}\left(y_{1}, y_{2}\right)\right) & \text { if } j=2,\end{cases}
\end{aligned}
$$

where

$$
j:= \begin{cases}1 & \text { if }\left(x_{1}, x_{2}, y_{1}, y_{2}\right) \in \bar{X}_{q}(1) \\ 2 & \text { if }\left(x_{1}, x_{2}, y_{1}, y_{2}\right) \in \bar{X}_{q}(2)\end{cases}
$$

The integers $k^{-}$and $k^{+}$are defined as above,

$$
\begin{aligned}
& k_{1}:= \begin{cases}k^{-} & \text {if } k^{-} \text {is even }, \\
1 & \text { if } k^{-} \text {is odd },\end{cases} \\
& k_{2}:= \begin{cases}k^{+} & \text {if } k^{+} \text {is even }, \\
k^{+}-1 & \text { if } k^{+} \text {is odd },\end{cases}
\end{aligned}
$$

and $A_{L}, A_{L}^{*}, A_{R}^{+}, A_{R}^{-}, C$ and $A_{i}$ are defined as in Section 7, in fine, replacing $k^{-}$by $k_{1}$ and $k^{+}$by $k_{2}$, respectively.

Define $j^{(t)}, k^{(t)}, A_{L}^{(t)}, A_{R}^{(t)}$ and $C^{(t)}$ as above. Note that, by construction $A_{R}^{(t)}$ and $C^{(t)}$ are integers, and $A_{R}^{(t)}>C^{(t)}$. An invariant measure can be found, although requiring a certain technical effort, using the method described in Section 6 . The inverse matrices of the algorithm are given by

$$
\beta_{q}^{(t)}(1)=\left(\begin{array}{ccc}
A_{L}^{(t)} & 1 & 0 \\
\frac{1}{A_{R}^{(t-1)}} & 0 & 0 \\
0 & 0 & 1
\end{array}\right), \quad \beta_{q}^{(t)}(2)=\left(\begin{array}{ccc}
A_{L}^{(t)} A_{R}^{(t)} & 0 & 1 \\
\frac{1}{A_{R}^{(t)}} A_{R}^{(t)} & 0 & 0 \\
C^{(t)} & 1 & 0
\end{array}\right),
$$

where $A_{R}^{(t-1)}=1$ if $j^{(t-1)}=1$. The convergence matrices, and thus the sequences of Diophantine approximations, are defined as above. To estimate the exponent of convergence, we use the modified method of Paley and Ursell [17], as described in Schweiger [22]. It is based on the following quantities:

Definition. For $i=1,2$, set

$$
[t, s]:=q^{(t)} p_{i}^{(s)}-q^{(s)} p_{i}^{(t)}
$$

and

$$
\rho_{t+3}:= \begin{cases}\max \left\{\frac{[t+3, t+2]}{q^{(t+3)}}, \frac{[t+3, t]}{\left.q^{(t+3)}\right\}}\right. & \text { if } j^{(t+2)}=1 \\ \max \left\{\frac{[t+3, t+2]}{q^{(t+3)}}, \frac{[t+3, t+1]}{\left.q^{(t+3)}\right\}}\right. & \text { if } j^{(t+2)}=2 .\end{cases}
$$


It is known that

$$
\left|x_{i}-\frac{p_{i}^{(t)}}{q^{(t)}}\right| \leq \frac{2 \rho_{t}}{q^{(t)}}
$$

(see e.g. [18]), thus exponential decay of $\rho_{t}$ yields exponential convergence to $\left(x_{1}, x_{2}\right)$. We have the following recursion relations (since the results hold for both $p_{1}^{(.)}$and $p_{2}^{(.)}$, we write $p^{(.)}$instead):

if $j^{(t+2)}=1$ :

$$
q^{(t+4)}=A_{L}^{(t+4)} A_{R}^{(t+4)} q^{(t+3)}+\frac{A_{R}^{(t+4)}}{A_{R}^{(t+3)}} q^{(t)}+C^{(t+4)} q^{(t+2)},
$$

if $j^{(t+3)}=1$ :

$$
q^{(t+4)}=A_{L}^{(t+4)} A_{R}^{(t+4)} q^{(t+3)}+A_{R}^{(t+4)} q^{(t+2)}+C^{(t+4)} q^{(t+1)}
$$

if $j^{(t+2)}=j^{(t+3)}=2$ :

$$
q^{(t+4)}=A_{L}^{(t+4)} A_{R}^{(t+4)} q^{(t+3)}+\frac{A_{R}^{(t+4)}}{A_{R}^{(t+3)}} q^{(t+1)}+C^{(t+4)} q^{(t+2)}
$$

if $j^{(t+2)}=1$ :

$$
\begin{aligned}
& {[t+4, t+3]=-\frac{A_{R}^{(t+4)}}{A_{R}^{(t+3)}}[t+3, t]-C^{(t+4)}[t+3, t+2],} \\
& {[t+4, t+2]=A_{L}^{(t+4)} A_{R}^{(t+4)}[t+3, t+2]-\frac{A_{R}^{(t+4)}}{A_{R}^{(t+3)}}[t+2, t],}
\end{aligned}
$$

if $j^{(t+3)}=1$ :

$$
\begin{aligned}
& {[t+4, t+3]=-A_{R}^{(t+4)}[t+3, t+2]-C^{(t+4)}[t+3, t+1],} \\
& {[t+4, t+1]=A_{L}^{(t+4)} A_{R}^{(t+4)}[t+3, t+1]+A_{R}^{(t+4)}[t+2, t+1],}
\end{aligned}
$$

if $j^{(t+2)}=j^{(t+3)}=2$ :

$$
\begin{aligned}
& {[t+4, t+3]=-\frac{A_{R}^{(t+4)}}{A_{R}^{(t+3)}}[t+3, t+1]-C^{(t+4)}[t+3, t+2],} \\
& {[t+4, t+2]=A_{L}^{(t+4)} A_{R}^{(t+4)}[t+3, t+2]-\frac{A_{R}^{(t+4)}}{A_{R}^{(t+3)}}[t+2, t+1] .}
\end{aligned}
$$

From these relations, we deduce the following 


\section{Lemma 8.1.}

$$
|[t+4, t+3]| \leq\left(q^{(t+4)}-q^{(t)}\right) \max \left\{\rho_{t+3}, \rho_{t+2}, \rho_{t+1}\right\}
$$

Proof. We only show the cyclic case $j^{(t+1)}=j^{(t+2)}=j^{(t+3)}=2$. The other cases are similar. We use the above recursion relations. If $C^{(t+4)}=0$, then $A_{R}^{(t+4)}=1$. We get

$$
\begin{aligned}
|[t+4, t+3]| & =\left|-\frac{1}{A_{R}^{(t+3)}}[t+3, t+1]\right| \\
& \leq \frac{1}{A_{R}^{(t+3)}} q^{(t+3)} \rho_{t+3} \\
& \leq\left(q^{(t+4)}-\left(\left(A_{L}^{(t+4)}-\frac{1}{A_{R}^{(t+3)}}\right) q^{(t+3)}+\frac{1}{A_{R}^{(t+3)}} q^{(t+1)}\right)\right) \rho_{t+3} \\
& \leq\left(q^{(t+4)}-q^{(t+1)}\right) \rho_{t+3} .
\end{aligned}
$$

Now let $C^{(t+4)} \geq 1$, hence $A_{R}^{(t+4)} \geq 2$. If $[t+3, t+1][t+3, t+2] \leq 0$, then

$$
\begin{aligned}
|[t+4, t+3]| & =\left|-\frac{A_{R}^{(t+4)}}{A_{R}^{(t+3)}}[t+3, t+1]-C^{(t+4)}[t+3, t+2]\right| \\
& \leq A_{R}^{(t+4)} q^{(t+3)} \rho_{t+3} \\
& \leq\left(q^{(t+4)}-q^{(t+2)}\right) \rho_{t+3} .
\end{aligned}
$$

If $[t+3, t+1][t+3, t+2] \geq 0, C^{(t+3)}=0$ and $A_{R}^{(t+3)}=1$, then

$$
\begin{aligned}
|[t+4, t+3]| & =\left|-\frac{A_{R}^{(t+4)}}{A_{R}^{(t+3)}}[t+3, t+1]-C^{(t+4)}[t+3, t+2]\right| \\
& =\left|-A_{R}^{(t+4)}[t+3, t+1]+\frac{C^{(t+4)}}{A_{R}^{(t+2)}}[t+2, t]\right| \\
& \leq\left(A_{R}^{(t+4)} q^{(t+3)}+C^{(t+4)} q^{(t+2)}\right) \max \left\{\rho_{t+3}, \rho_{t+2}\right\} \\
& \leq\left(q^{(t+4)}-q^{(t+1)}\right) \max \left\{\rho_{t+3}, \rho_{t+2}\right\} .
\end{aligned}
$$

If $[t+3, t+1][t+3, t+2] \geq 0, C^{(t+3)} \geq 1$, and thus $A_{R}^{(t+3)} \geq 2$, we have two cases: $[t+2, t][t+2, t+1] \leq 0$ yields 


$$
\begin{aligned}
|[t+4, t+3]|= & \left|-\frac{A_{R}^{(t+4)}}{A_{R}^{(t+3)}}[t+3, t+1]-C^{(t+4)}[t+3, t+2]\right| \\
= & \mid-A_{L}^{(t+3)} A_{R}^{(t+4)}[t+2, t+1]+\frac{A_{R}^{(t+4)}}{A_{R}^{(t+2)}}[t+1, t] \\
& +\frac{A_{R}^{(t+3)} C^{(t+4)}}{A_{R}^{(t+2)}}[t+2, t]+C^{(t+4)} C^{(t+3)}[t+2, t+1] \mid \\
\leq & \left(\left(A_{L}^{(t+3)} A_{R}^{(t+4)} A_{R}^{(t+3)}-1\right) q^{(t+2)}+A_{R}^{(t+4)} q^{(t+1)}\right. \\
& \left.+C^{(t+4)} q^{(t+2)}\right) \max \left\{\rho_{t+2}, \rho_{t+1}\right\} \\
\leq & \left(q^{(t+4)}-q^{(t+2)}\right) \max \left\{\rho_{t+2}, \rho_{t+1}\right\},
\end{aligned}
$$

while if $[t+2, t][t+2, t+1] \geq 0$, then $[t+3, t+2][t+2, t+1] \leq 0$ and

$$
\begin{aligned}
|[t+4, t+3]|=\left|-\frac{A_{R}^{(t+4)}}{A_{R}^{(t+3)}}[t+3, t+1]-C^{(t+4)}[t+3, t+2]\right| \\
=\mid-A_{L}^{(t+3)} A_{R}^{(t+4)}[t+2, t+1] \\
\quad+\frac{A_{R}^{(t+4)}}{A_{R}^{(t+2)}}[t+1, t]-C^{(t+4)}[t+3, t+2] \mid \\
\leq\left(A_{R}^{(t+4)} q^{(t+3)}+\frac{A_{R}^{(t+4)}}{A_{R}^{(t+3)}} q^{(t+1)}\right) \max \left\{\rho_{t+3}, \rho_{t+2}, \rho_{t+1}\right\} \\
\leq\left(q^{(t+4)}-q^{(t+2)}\right) \max \left\{\rho_{t+3}, \rho_{t+2}, \rho_{t+1}\right\} .
\end{aligned}
$$

Similarly, we have

Lemma 8.2. Let $j^{(t+3)}=2$, then

$$
|[t+4, t+2]| \leq\left(q^{(t+4)}-q^{(t)}\right) \max \left\{\rho_{t+3}, \rho_{t+2}, \rho_{t+1}, \rho_{t}\right\}
$$

Lemma 8.3. Let $j^{(t+4)}=1$, then

$$
|[t+5, t+2]| \leq\left(q^{(t+5)}-q^{(t)}\right) \max \left\{\rho_{t+3}, \rho_{t+2}, \rho_{t+1}, \rho_{t}\right\} .
$$

Now define $\tau_{t}:=\max \left\{\rho_{t+4}, \rho_{t+3}, \rho_{t+2}, \rho_{t+1}, \rho_{t}\right\}$. Using Lemmata 8.1 8.3 and the above definitions, we estimate

$$
\tau_{t+5} \leq\left(1-\min \left\{\frac{q^{(t)}}{q^{(t+5)}}, \frac{q^{(t+1)}}{q^{(t+6)}}, \frac{q^{(t+2)}}{q^{(t+7)}}, \frac{q^{(t+3)}}{q^{(t+8)}}, \frac{q^{(t+4)}}{q^{(t+9)}}\right\}\right) \tau_{t}
$$


Since

$$
\frac{q^{(t)}}{q^{(t+5)}}>0
$$

almost everywhere, we may define a function

$$
g\left(x_{1}, x_{2}, y_{1}, y_{2}\right):=\log \left(1-\min \left\{\frac{q^{(1)}}{q^{(6)}}, \frac{q^{(2)}}{q^{(7)}}, \frac{q^{(3)}}{q^{(8)}}, \frac{q^{(4)}}{q^{(9)}}, \frac{q^{(5)}}{q^{(10)}}\right\}\right)
$$

to apply the ergodic theorem

$$
\lim _{t \rightarrow \infty} \sum_{s=0}^{t-1} g\left(\bar{T}_{q}^{s}\left(x_{1}, x_{2}, y_{1}, y_{2}\right)\right)=\int_{\bar{X}_{q}} g\left(x_{1}, x_{2}, y_{1}, y_{2}\right) d \mu=: \log K<0
$$

almost everywhere. Thus $\tau_{t} \leq c K^{\frac{t}{5}}$, and $\rho_{t}$ goes down exponentially. We state the following

Theorem 8.4. For the algorithm $\overline{T_{q}}$, there exists a constant $d_{q}$ such that, for almost all $\left(x_{1}, x_{2}, y_{1}, y_{2}\right)$ in $\bar{X}_{q}$, there exist an integer $t\left(x_{1}, x_{2}, y_{1}, y_{2}\right)$, such that the inequality

$$
\left|x_{i}-\frac{p_{i}^{(t)}}{q^{(t)}}\right| \leq \frac{1}{\left(q^{(t)}\right)^{1+d_{q}}} .
$$

hold for any $t \geq t\left(x_{1}, x_{2}, y_{1}, y_{2}\right)$.

Remark. Exponential convergence of $\bar{T}_{q}$ follows directly from exponential convergence of the multiplicative acceleration of Brun's Algorithm. However, the proof of Theorem 8.4 is interesting for a different reason: Hitherto, proofs for exponential convergence of Brun's algorithm were based on considering a special subset of $X_{B}$ i.e., the set where $j^{(1)}=\cdots=j^{(t)}=2$ for some $t \geq 3$, and the induced transformation on this set (compare R. Meester [12] or [18]). We may now (with respect to the measure of the singularization area $S_{q}$ ) transfer the above result, especially the estimate of the decay using the function $g\left(x_{1}, x_{2}, y_{1}, y_{2}\right)$, to the multiplicative acceleration of Brun's algorithm using standard techniques, which essentially were described in the original work of Paley and Ursell [17]. We immediately see that not only these special sets, but all cylinders contribute to the exponential approximation. However, the estimate of the approximation speed depends on the size of the quantities $q^{(t+5)}-q^{(t)}$. The smaller this difference, the better the estimate. Thus the estimate gets worse if a large number of non-cyclic convergents with suitable partial quotients has been singularized, which essentially leads to the counterexample for cyclic algorithms in [17]. 


\section{Acknowledgements}

This paper is part of research done in connection with the project P15132 (Multidimensional Continued Fractions) of the Austrian Science Foundation FWF. I would especially like to thank Prof. F. Schweiger for numerous valuable suggestions and comments. I also want to thank the anonymous referee for his careful revision, which considerably improved the exposition of the paper.

\section{References}

[1] P. ARnoux, A. Nogueira, Mesures de Gauss pour des algorithmes de fractions continues multidimensionnelles. Ann. Sci. Ec. Norm. Supér., IV. Sér. 26 (1993), 645-664.

[2] W. Bosma, Optimal continued fractions, Indag. Math. 49 (1987), 353-379.

[3] W. Bosma, H. JAGER, F. WIEDIJK, Some metrical observations on the approximation by continued fractions. Indag. Math. 45 (1983), 281-299.

[4] V. BRuN, Algorithmes euclidiens pour trois et quatre nombres. 13 Skand Mat-Kongr, Helsinki (1957), 45-64.

[5] K. DAJANI, C. KRAAIKAMP, The mother of all continued fractions. Colloq. Math. 84-85 (2000), Pt. 1, 109-123.

[6] A. HuRwITz, Über eine besondere Art des Kettenbruch-Entwicklung reeller Grössen, Acta Math. 12 (1889), 367-405.

[7] M. Iosifescu, C. KRAAIKAMP, Metrical Theory of Continued Fractions. Kluwer Academic Publishers, Dordrecht (2002).

[8] S. ITO, Algorithms with mediant convergents and their metrical theory. Osaka J. Math. 26 (1989), No.3, 557-578.

[9] C. KRAAIKAMP, Metric and Arithmetic Results for Continued Fraction Expansions, Academic Thesis, Univ. Amsterdam (1991), pp. 185.

[10] C. KRAAIKAMP, A new class of continued fraction expansions.. Acta Arith. 57 (1991), 1-39.

[11] J. LEHNER, Semiregular continued fractions whose partial denominators are 1 or 2 . In: The Mathematical Legacy of Wilhelm Magnus: Groups, Geometry and Special Functions (Brooklyn, NY, 1992), pp. 407-410. Contemporary Mathematics 169, Amer. Math. Soc., Providence, RI 169 (1992), 407-410.

[12] R. MEester, A simple proof of the exponential convergence of the modified Jacobi-Perron algorithm. Erg. Th. Dyn. Sys. 19 (1999), 1077-1083.

[13] H. MinkowsKI, Über dis Annäherung an eine reelle Grösse durch rationale Zahlen, Math. Ann., 54 (1901), 91-124.

[14] B. Minnigerode, Über eine neue Methode, die Pell'sche Gleichung aufzulösen, Nachr. König. Gesellsch. Wiss. Göttingen Math.-Phys. Kl. 23 (1873), 619-652.

[15] H. NAKADA, Metrical theory for a class of continued fraction transformations and their natural extensions. Tokyo J. Math. 4 (1981), 399-426.

[16] H. NAKADA, S. ITO, S. TANAKA, On the invariant measure for the transformations associated with some real continued fractions. Keio Eng. Rep. 30 (1977), 159-175.

[17] R.E.A.C. Paley, H.B. Ursell, Continued Fractions in Several Dimensions. Proc. Camb. Phil. Soc. 26 (1930), 127-144.

[18] B.R. Schratzberger, The Exponent of Convergence for Brun's Algorithm in two Dimensions. SBer. Österr. Akad. Wiss. Math.-naturw. Kl. Abt. II, 207 (1998), 229-238.

[19] B.R. Schratzberger, On the Singularization of the two-dimensional Jacobi-Perron Algorithm. Preprint Universität Salzburg.

[20] F. SCHWEIGER, Invariant measures for maps of continued fraction type. J. Number Theory 39 (1991), No.2, 162-174.

[21] F. SchweIGer, Ergodic Theory of Fibred Systems and Metric Number Theory. Clarendon Press, Oxford (1995). 
[22] F. SCHWEIGER, The exponent of convergence for the 2-dimensional Jacobi-Perron Algorithm. Nowak, W.G. \& Schoissengeier, J. (eds), Proceedings of the Conference of Analytic and Elementary Number Theory Vienna, 207-213.

[23] F. Schweiger, Multidimensional Continued Fractions. Univ Press, Oxford (2000).

Bernhard SCHRATZBERGER

Universität Salzburg

Institut für Mathematik

Hellbrunnerstraße 34

5020 Salzburg, Austria

E-mail : bernhard.schratzberger@sbg.ac. at 